ARTICLE

\title{
Tipping the immunostimulatory and inhibitory DAMP balance to harness immunogenic cell death
}

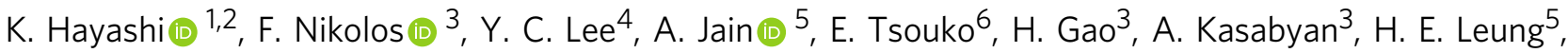 \\ A. Osipov ${ }^{3}$, S. Y. Jung (10 ${ }^{5}$, A. V. Kurtova ${ }^{2} \&$ K. S. Chan ${ }^{1,3 凶}$
}

Induction of tumor cell death is the therapeutic goal for most anticancer drugs. Yet, a mode of drug-induced cell death, known as immunogenic cell death (ICD), can propagate antitumoral immunity to augment therapeutic efficacy. Currently, the molecular hallmark of ICD features the release of damage-associated molecular patterns (DAMPs) by dying cancer cells. Here, we show that gemcitabine, a standard chemotherapy for various solid tumors, triggers hallmark immunostimualtory DAMP release (e.g., calreticulin, HSP70, and HMGB1); however, is unable to induce ICD. Mechanistic studies reveal gemcitabine concurrently triggers prostaglandin $E_{2}$ release as an inhibitory DAMP to counterpoise the adjuvanticity of immunostimulatory DAMPs. Pharmacological blockade of prostaglandin $E_{2}$ biosythesis favors $\mathrm{CD}_{103}{ }^{+}$dendritic cell activation that primes a $\mathrm{Tc} 1$-polarized $\mathrm{CD} 8^{+} \mathrm{T}$ cell response to bolster tumor rejection. Herein, we postulate that an intricate balance between immunostimulatory and inhibitory DAMPs could determine the outcome of drug-induced ICD and pose COX-2/ prostaglandin $E_{2}$ blockade as a strategy to harness ICD.

\footnotetext{
${ }^{1}$ Department of Pathology and Laboratory Medicine, Cedars-Sinai Medical Center, Los Angeles, CA 90048, USA. ${ }^{2}$ Graduate Program in Translational Biology and Molecular Medicine, Baylor College of Medicine, Houston, TX 77030, USA. ${ }^{3}$ Samuel Oschin Cancer Center, Cedars-Sinai Medical Center, Los Angeles, CA 90048, USA. ${ }^{4}$ Graduate Institute of Medical Sciences, Taipei Medical University, Taipei City, Taiwan. ${ }^{5}$ Alkek Center for Molecular Discovery, Proteomics Core, Baylor College of Medicine, Houston, TX 77030, USA. ${ }^{6}$ Department of Orthopedic Surgery, Baylor College of Medicine, Houston, TX 77030, USA. ${ }^{\bowtie}$ email: keithsyson.chan@cshs.org
} 
M ost anticancer therapies designate tumor cell death as the ultimate biological and therapeutic end goal. Yet, studies are emerging to illuminate the often-overlooked physiological processes resulting from cell death as important factors that influence therapeutic efficacy. During development and homeostasis, billions of cells undergo a highly orchestrated mode of immunologically silent (or tolerogenic) cell death-these dead/dying cells are cleared by innate immune cells without provoking an inflammatory response. In contrast, cells that undergo immunogenic cell death (ICD) during pathogenic or xenobiotic insult, for example, actively potentiate an adaptive immune response to properly resolve these pathologic conditions ${ }^{1-6}$. In the context of anticancer therapeutics, drugs capable of provoking ICD are deemed clinically relevant based on their inherent propensity to augment therapeutic efficacy via recruiting antitumoral immunity ${ }^{1-6}$.

The current established paradigm and defining molecular hallmark of ICD is the release of host-derived, immune-activating molecules known as damage-associated molecular patterns (DAMPs) from dying cells ${ }^{1-7}$. Canonical DAMPs include, but are not limited to: calreticulin $(\mathrm{CRT})^{8}$; heat-shock protein $70 \mathrm{kDa}$ $(\mathrm{HSP} 70)^{9}$; and high-mobility group box 1 (HMGB1) ${ }^{10}$. During the initial stages of ICD induction, CRT translocates from the endoplasmic reticulum (ER) to the cell surface and functions as an "eat-me" signal" ${ }^{11}$. The translocation of HSP70 from the ER to cell-surface during mid-apoptosis 9 and the extracellular release of HMGB1 during late-apoptosis ${ }^{10}$ serve as host-derived "danger" signals (or immunological adjuvants ${ }^{3}$ ). These ectopically expressed DAMPs are postulated to function as signal 0 to engage pattern recognition receptors on professional antigen-presenting cells (e.g., dendritic cells ${ }^{11}$ ) and other innate immune cells ${ }^{12}$. Together with signal 1 ( $\mathrm{T}$ cell receptor: major histocompatibility complex), signal 2 (co-stimulation), and signal 3 (cytokine), these professional antigen-presenting cells are properly matured and activated-priming an effective $\mathrm{CD}^{+}{ }^{+} \mathrm{T}$ cell immune response ${ }^{8-10,13-15}$.

Clinically, drug-induced immunogenic cell death is reported to positively correlate with therapeutic response and associate with an enhanced antitumoral $\mathrm{CD}^{+} \mathrm{T}$ cell immunity ${ }^{16-20}$. To date, only a handful of anticancer agents are recognized as bona fide ICD-inducers ${ }^{17,21}$; most therapeutics require the assistance of adjuvant drugs to substantiate DAMP release for successful ICD induction $^{22}$. Thus, in contrast to bona fide ICD-inducers, such as anthracyclines (e.g., mitoxantrone $)^{23-27}$, most standard-of-care chemotherapies, including cisplatin ${ }^{17}$ and gemcitabine ${ }^{28}$, are considered non-ICD-inducing as monotherapies. The immunological adjuvanticity of hallmark DAMPs in propagating antitumoral immune response(s) have been extensively studied ${ }^{1-11}$. However, whether chemotherapy-induced DAMP release alone dictates the immunogenic versus tolerogenic fate of cell death is not fully understood.

Here, we pose the induction of prostaglandin $E_{2}$ as an interregulatory counterpoise to immunostimulatory DAMPs in the context of chemotherapy-induced ICD. Albeit the success of gemcitabine in inducing hallmark DAMP release, $\mathrm{PGE}_{2}$ dominantly skews cell death towards a tolerogenic, rather than immunogenic phenotype. Building on the prior works of others, we define $\mathrm{PGE}_{2}$ as an inhibitory DAMP (iDAMP) that converges with immunostimulatory DAMPs as signal 0 to dictate the maturation of dendritic cells and influence CD8 ${ }^{+} \mathrm{T}$ cell effector function.

\section{Results}

Gemcitabine potentiates the release of hallmark DAMPs from dying cancer cells. Most chemotherapeutic agents are nonimmunogenic, with only a handful reported to be ICD-inducing 4 . These immunogenic chemotherapies are characterized by their capacity to induce dying cells that (i) are coupled with hallmark DAMP release ${ }^{3}$ and (ii) serve as functional in vivo vaccines to prime an antitumoral $\mathrm{CD}^{+} \mathrm{T}$ cell response using a goldstandard vaccination assay ${ }^{26,27}$. Gemcitabine is a commonly prescribed chemotherapy for treating various solid tumors (e.g., bladder and pancreatic cancers). However, whether gemcitabine as a monotherapy potentiates ICD via hallmark DAMP release is limited; a prior study reported that gemcitabine is nonimmunogenic ${ }^{28}$. To evaluate this further, we subjected human bladder cancer cells (i.e., T24) to gemcitabine chemotherapy treatment in vitro. Quantitative proteomic profiling of fractionated cellular compartments via mass spectrometry was performed to identify cell surface and extracellularly secreted proteins upon gemcitabine treatment. To categorize relevant proteins, we employed two analytical approaches: (i) proteins sharing similar amino acid sequences, protein domains, or structures and (ii) proteins sharing similar functions. While no structurally similar proteins were revealed by mass spectrometry, we detected cell surface calreticulin (CRT; Fig. 1a, b) and other

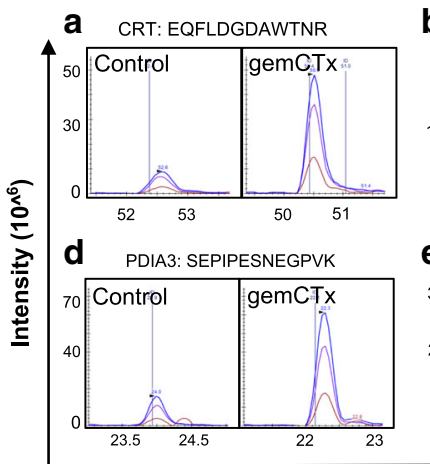

b HSP70: LEDTENWLYEDGEDQPK
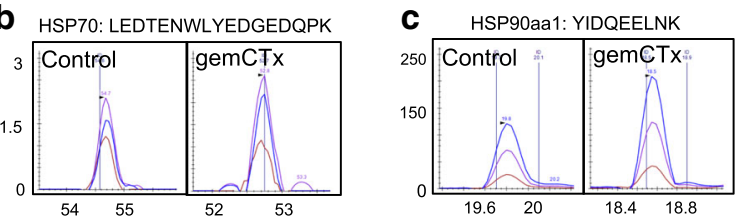

e ANXA1: TPAQFDADELR

f HMGB1: LGEMWNNTAADDKQPYEK
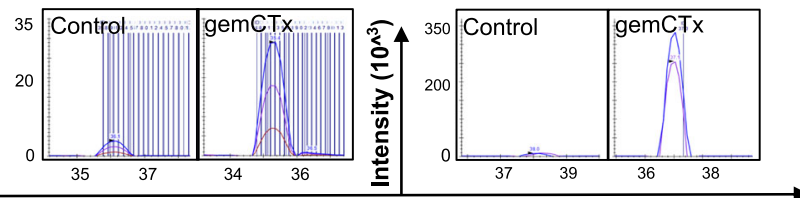

Retention time

Fig. 1 The release of hallmark DAMPs from gemcitabine-induced dying cancer cells. Representative peptide chromatogram illustrating differential enrichment of a CRT, b HSP70, c HSP90aa1, and d PDIA3 on the cell surface, as well as e ANXA and $\mathbf{f}$ HMGB1 released into the cultured media by cancer cells (murine $\mathrm{G} 69$ bladder cancer cells) treated with gemcitabine ( $n=2$ independent mass spectrometry proteomic profiling experiments). ANXA1 annexin A1, CRT calreticulin, gemCTx gemcitabine chemotherapy-treated, HMGB1 high-mobility group protein box 1, HSP heat-shock protein, PDIA3 protein disulfide isomerase A3. Extracted chromatograms for a representative peptide of each protein was plotted using the Skyline software; precursor (blue color), precursor $[M+1]$ (purple color), precursor $[M+2]$ (red color) represent the three isotopic peaks for each indicated peptide. 
Table 1 Mass Spectrometry profiling reveals hallmark DAMPs from dying cancer cells after gemcitabine treatment.

\begin{tabular}{llccc} 
Protein name & $\begin{array}{l}\text { T24 gemCTx:control } \\
\text { (unique peptide no.) }\end{array}$ & $\begin{array}{l}\text { G69 gemCTx:control } \\
\text { (iFOT ratio) }\end{array}$ & $\begin{array}{l}\text { Panc02 gemCTx:control } \\
\text { (iFOT ratio) }\end{array}$ & Fraction analyzed \\
\hline ANXA1 & ND & $606: 285(2.12)$ & $33.15:$ ND $(33.15)$ & Cultured media \\
CRT & 10 & $108: 14.8(7.29)$ & $243: 16.4(14.82)$ & Cell membrane \\
HMGP HMGB1 & 2 & $13.2: 7.4(1.78)$ & $17.3:$ ND $(17.3)$ & Cultured media \\
HSP70 & 8 & $5.3: 3.1(1.71)$ & $14.3: 4.5(3.18)$ & Cell membrane \\
HSP90 & 29 & $142: 103(1.38)$ & $138.5: 93.6(1.48)$ & Cell membrane \\
PDIA3 & ND & $147: 28.5(5.16)$ & $247.5: 12.2(20.29)$ & Cell membrane \\
\hline
\end{tabular}

A table summarizing the detection of classical DAMPs from gemcitabine-induced dying cancer cells, via mass spectrometry (MS) proteomic profiling. Quantification of peptide enrichment (gemCTx- vs. non-treated control) by either (i) number of unique peptide sequences (T24 cells) or (ii) average iFOT ratio (G69 and Panc02 cells; $n=2$ independent experiments) for each of the detected proteins. ANXA1 annexin A1, CRT calreticulin, gemCTx gemcitabine chemotherapy-treated, HMGB1 high-mobility group protein box 1, HSP heat-shock protein, ND not detected, PDIA3 protein disulfide isomerase $A 3$.

proteins with known immunological effector function $(s)^{3}$. Intriguingly, a series of proteins classified as hallmark DAMPs were identified in the extensive mass spectrometry protein list. These included cell-surface heat-shock proteins $70 \mathrm{kDa}$ (HSP70) and 90 $\mathrm{kDa}$ (HSP90), as well as extracellular high-mobility group protein (HMGP; Fig. 1a). Specific protein enrichment was quantified by grouping unique peptide sequences corresponding to each DAMP and compared to gemcitabine-treated versus untreated cells (Table 1 and Supplementary Fig. 1a). These initial findings challenged the prior study reporting that gemcitabine is unable to promote cell surface DAMP (i.e., CRT) expression to induce ICD in a pancreatic ductal adenocarcinoma (PDAC) model $^{28}$.

Since T24 is a human bladder cancer cell line and does not allow for the functional evaluation of ICD in immunocompetent hosts, we developed a murine bladder cancer model (designated hereinafter as G69). The advent of this tool permits for the functional evaluation of bladder cancer ICD in syngeneic, immunocompetent hosts in vivo (Supplementary Fig. 2). As a complementary approach, we also utilized a murine PDAC model that was previously established for investigating ICD (i.e., Panc $02^{28}$ ), as proof-of-concept to generalize our findings in a different tumor type. Proteomic profiling was performed using the cell-surface fraction and cultured medium of gemcitabine- or vehicle-treated G69 and Panc02 cells in vitro (Table 1). As demonstrated in Fig. 1a-e, in addition to the enriched expression of cell surface CRT, HSP70, and HSP90, gemcitabine treatment also induced the expression of disulfide isomerase family A member 3 (PDIA3) ${ }^{29}$, as well as the extracellular release of highmobility group protein B1 (HMGB1) and annexin A1 (ANXA1) ${ }^{30}$ into the cultured media (Fig. 1e-g and Supplementary Fig. 1b). Here, proteomic enrichment of DAMPs was quantified using the total unique protein counts normalized to total fraction counts and unique peptide sequences (iFOT; Fig. 1a; additional representative peptide peaks are presented as raw data in Supplementary Fig. 1). Collectively, these profiling results from three independent cancer models convincingly demonstrated that gemcitabine, as a monotherapy, potentiates hallmark DAMP release as a generalized phenomenon-the current molecular prerequisite of ICD.

Hallmark DAMP release is insufficient to induce immunogenic cell death. To validate the mass spectrometry results in Fig. 1, we profiled for cell surface and extracellular DAMPs using flow cytometry and western blot, respectively. Reflective of the proteomics analyses, both human T24 and murine G69 bladder cancer cells displayed enrichment of cell surface CRT and HSP70 after $48 \mathrm{~h}$ of gemcitabine treatment in vitro, when compared to vehicle-treated control cells (Fig. 2a). Importantly, the cell surface expression of CRT was present in DAPI-negative, membrane impermeable (i.e., live) cells (Supplementary Fig. 3a-c). While the bona fide ICD-inducing chemotherapy, mitoxantrone (anthracycline ${ }^{24}$ ), potentiated substantial cell surface CRT expression in all three models, cisplatin (a non-ICD-inducing chemotherapy), in contrast, failed to promote significant cell surface CRT expression $^{8,24}$ (Supplementary Fig. 3a-c). These results further corroborate the previous works of others, demonstrating the inadequacy of cisplatin to promote cell surface CRT translocation $^{8,23}$. As for extracellular DAMPs, western blot analyses confirmed the release of HMGB1 into the culture media by both G69 and Panc02 cancer cells treated with gemcitabine for 48 h (Fig. 2b and Supplementery Fig. 3d, e). Additionally, gemcitabine treatment also prompted the release of a non-protein extracellular DAMP, ATP 25 (Supplementary Fig. 3f, g).

The release of both "eat-me" and "danger" DAMP signals from multiple cancer models led us to re-evaluate the immunogenic properties of gemcitabine using the gold-standard in vivo vaccination assay 26,27 . First, murine G69 cancer cells were treated in vitro with IC-50 dose of chemotherapy for $24 \mathrm{~h}$ to initiate cell death. Next, either dying cells exposed to mitoxantrone (positive control) ${ }^{20}$, cisplatin (negative control) $)^{8,23}$, or gemcitabine (unknown), were injected subcutaneously into age-matched syngeneic hosts as vaccines in the left hind flank $(n=3$ mice per vaccine group). PBS was used as the non-treated control vaccine. Seven days post-vaccination, mice were challenged with live G69 cells via subcutaneous inoculation on the opposing, right lower flank (Fig. 2e). Vaccination with cancer cells exposed to ICD-inducers (e.g., mitoxantrone ${ }^{20}$ ) are expected to successfully prevent tumor engraftment when challenged, by priming an antigen-specific CD8 ${ }^{+} \mathrm{T}$ cell response (Supplementary Fig. 4a). Conversely, mice vaccinated with cells exposed to non-ICDinducers (e.g., cisplatin ${ }^{8,23}$ ) are expected to fail in tumor rejection (Supplementary Fig. $4 \mathrm{~b}$ ). While the bona fide ICD-vaccine (i.e., mitoxantrone-treated G69 cells) successfully promoted tumor rejection in mice a week after, the vaccine utilizing cisplatin (negative control) failed (Fig. 2f, g). These results corroborate the previous works of others: in our bladder cancer model, mitoxantrone is a conserved ICD-inducer, while cisplatin remains a non-ICD-inducer. Altogether, these results validated G69 as a viable study model for investigating ICD in bladder cancer.

Despite the induction of hallmark DAMPs in vitro, mice vaccinated with gemcitabine-treated cancer cells failed to present signs of successful immunization upon challenge. All tumors engrafted became palpable within 28 days post challenge (Fig. 2 f, g). Moreover, there were no statistical difference in tumor-free survival (Fig. 2f), nor tumor volume (Fig. 2g) between the negative controls (i.e., cisplatin and PBS) and gemcitabine vaccine groups. Therefore, the induction of hallmark DAMPs by gemcitabine is not sufficient to induce ICD in a biological setting. 

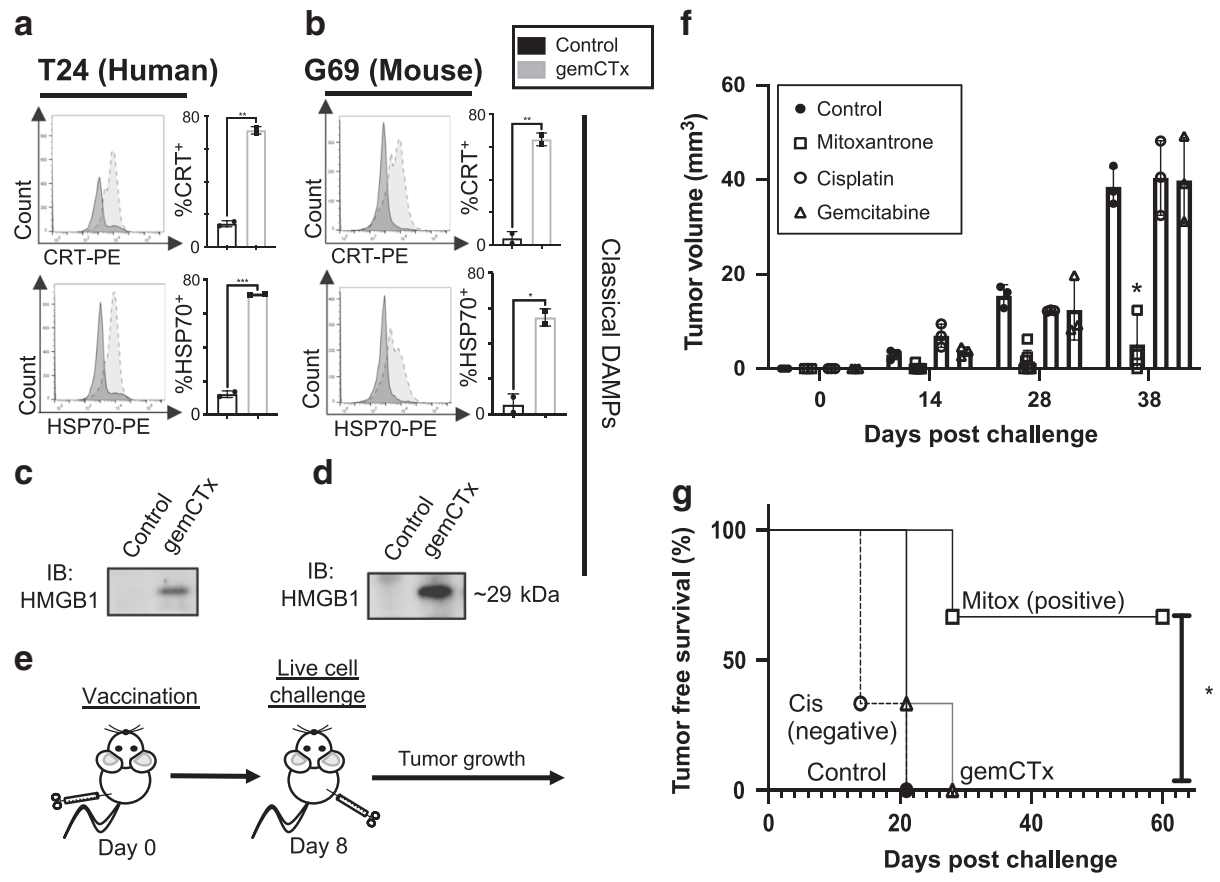

Fig. 2 Hallmark DAMP release is insufficient to induce immunogenic cell death. a, b Flow cytometry analysis and validation of DAMPs (i.e., CRT and HSP70) on the cell surface of human T24 and murine G69 bladder cancer cells treated with gemcitabine in vitro (representative plot shown with two technical replicates of $n=3$ independent experiments; example gating depicted in Supplementary Fig. 3). c, d Western blot of HMGB1, an extracellular DAMP, released into cultured media that were collected from gemcitabine-treated human T24 cells and murine G69 cells, respectively. e A schematic depiction of the in vivo vaccination assay treatment schedule for assaying immunogenic cell death. $\mathbf{f}$ Tumor volume and $\mathbf{g}$ corresponding tumor-free survival Kaplan-Meier plot ( $n=3$ mice per vaccination group; tumor volume calculation as indicated in Methods section) resulting from mice vaccinated with dying G69 cells via various chemotherapy and subsequently challenged with live G69 murine bladder cancer cells. cis cisplatin, gem gemcitabine, mitox mitoxantrone. Statistics: two-tailed, unpaired T test ( $\mathbf{a} ; p=0.0013$ [top] and $p=0.0006$ [bottom]) and $(\mathbf{b} ; p=0.0044$ [top] and $p=0.0123$ [bottom]); two-tailed, two-way ANOVA-Tukey's multiple comparisons test (f; $p<0.0240)$; Kaplan-Meier survival analysis using Mantel-Cox test (g; $p=0.0123)$; and where appropriate, data are presented as mean values \pm SEM.

Prostaglandin $\mathrm{E}_{2}$ release hinders ICD-induction by gemcitabine. The inability of the gemcitabine-treated G69 bladder cancer cells to potentiate proper immunization-despite hallmark DAMP release-led us to hypothesize that immunostimulatory DAMPs are unlikely the only molecular determinant governing immunogenic cell death ${ }^{3-5,31,32}$. A recent study has implicated prostaglandin $\mathrm{E}_{2}\left(\mathrm{PGE}_{2}\right)$ as an inhibitory DAMP (iDAMP) in the context of mechanical stress (i.e., repeated freezeand-thaw) using macrophages ${ }^{33}$. Independently, we have previously reported that gemcitabine-cisplatin chemotherapy induces cyclooxygenase-2 (COX-2) expression, resulting in the release of $\mathrm{PGE}_{2}$ from dying bladder tumor cells ${ }^{34}$. Therefore, we sought to investigate whether $\mathrm{PGE}_{2}$ could function as an iDAMP in this physiological response to chemotherapy treatment.

First, we investigated whether gemcitabine treatment alone potentiates the biosynthesis and release of $\mathrm{PGE}_{2}$ via upregulating COX-2 expression ${ }^{34}$. As shown, gemcitabine treatment of human T24 (Supplementary Fig. 5a) and murine G69 (Fig. 3a and Supplementary Fig. 5b) bladder cancer cells in vitro resulted in increased COX-2 expression, as well as significant $\mathrm{PGE}_{2}$ release into cultured media. Since both immunostimulatory DAMPs and iDAMP were released as a result of gemcitabine treatment, we hypothesized that the concurrent release of $\mathrm{PGE}_{2}$ counteracts the adjuvanticity of immunostimualtory DAMPs, and thus, prevent functional ICD.

To experimentally test this hypothesis, we utilized celecoxib drug treatment-a selective COX-2 molecular inhibitor-as a pharmacological approach to preclude $\mathrm{PGE}_{2}$ biosynthesis. We rationalized that the addition of celecoxib to gemcitabine treatment will alleviate the immunosuppressive constraints imposed by $\mathrm{PGE}_{2}$, and thereby, tip the balance in favor of immunostimulatory DAMPs to propagate an antitumoral response (Fig. 3b). To validate pharmacological COX-2/PGE blockade specifically targets the iDAMP without affecting immunostimulatory DAMPs, we treated murine G69 and Panc02 cancer cells with gemcitabine \pm celecoxib in vitro. Low dose celecoxib treatment did not significantly impact the rate of cell death (Supplemental Fig. 6a, b), expression of cell-surface CRT and HSP70, nor the extracellular release of HMGB1 into cultured media by both G69 (Fig. 3c and Supplementary Fig. 6c) and Panc02 (Fig. 3d and Supplementary Fig. 6c) cells. As expected, celecoxib significantly diminished the release of $\mathrm{PGE}_{2}$ into cultured media by both G69 (Fig. 3e and Supplementary Fig. 6d) and Panc02 cells (Fig. 3f and Supplementary Fig. 6d), when compared to gemcitabine-treated cells. These results indicate that pharmacological COX-2/PGE 2 blockade selectively inhibits iDAMP release without affecting immunostimulatory DAMP expression.

To evaluate the functional significance of iDAMP blockade in the context of ICD, we repeated the vaccination assay using G69 cells with the addition of a gemcitabine+celecoxib (i.e., gemCelex) vaccination arm. Mice were vaccinated with either PBS (non-treated control) or gemcitabine pre-treated cells \pm celecoxib drug treatment (test groups; $n=9$ mice per treatment arm). As expected, mice that were vaccinated with either PBS or gemcitabine-treated G69 cell vaccines developed palpable tumors within 28 days post challenge. Remarkably, mice that received the gemCelex (i.e., COX-2/PGE $\mathrm{P}_{2}$ blockade) vaccination displayed a significantly heightened antitumoral response, with hindered tumor growth (Fig. 3g; $p<0.05$ post 28 days) and engraftment 
a

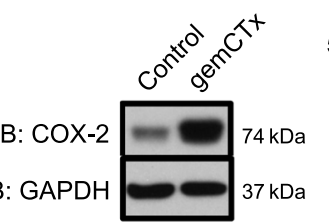

b
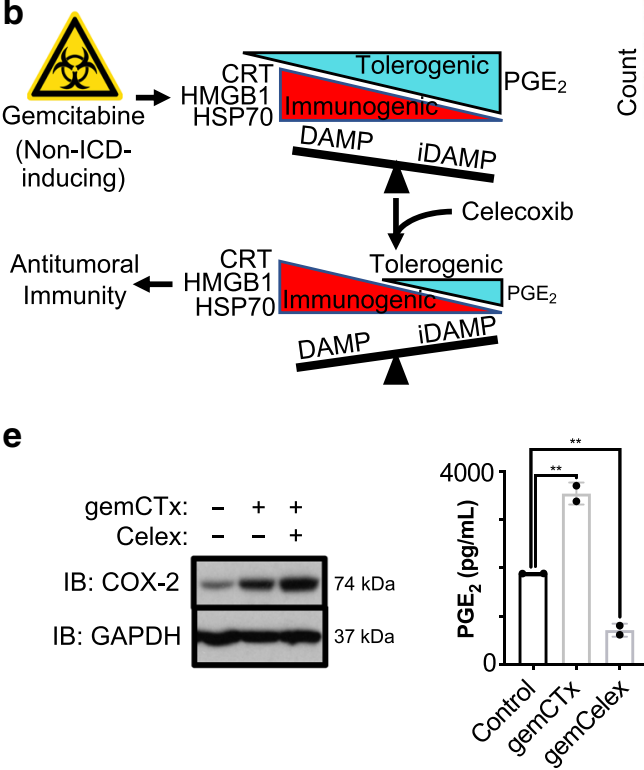

C

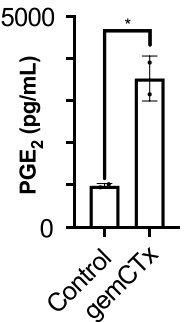

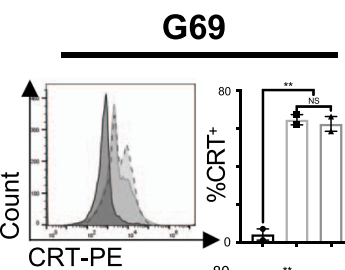

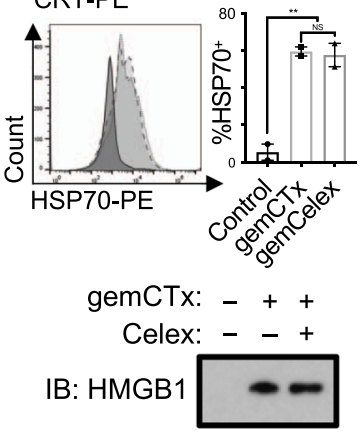

f

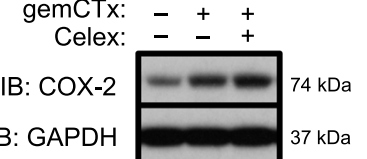

IB: GAPDH $37 \mathrm{kDa}$

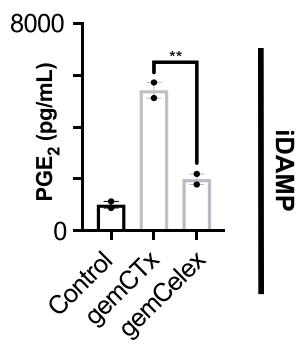

d

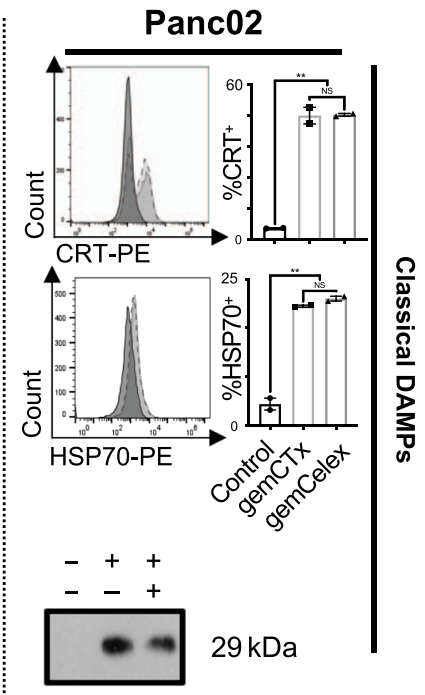

$\frac{0}{0}$
0
0
0
0
0
0
0
3
0
0
0
0 g

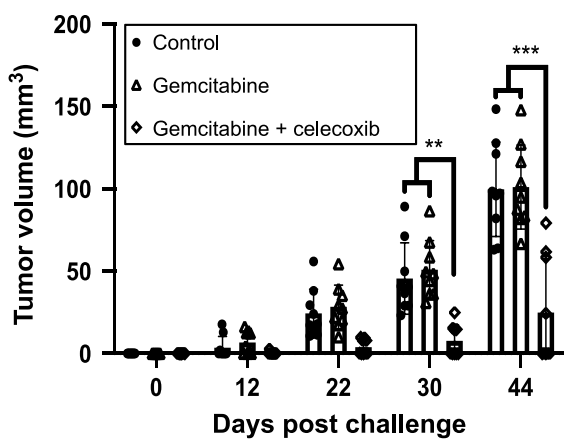

h

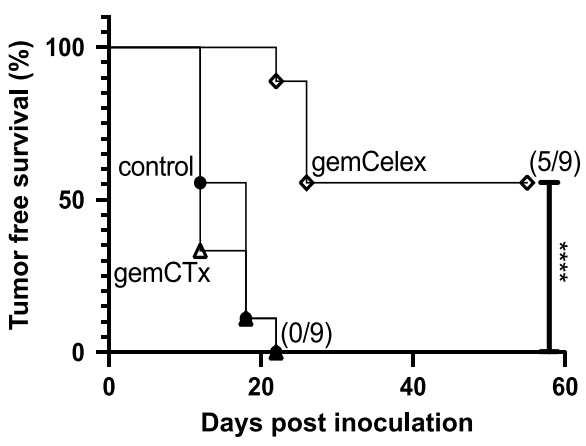

(Fig. 3h; $p<0.0001$ post 50 days). Next, we interrogated whether the effects of COX-2/PGE 2 blockade (Fig. $3 \mathrm{~g}, \mathrm{~h}$ ) is recapitulated in Panc02 cells using the same vaccination assay. In Panc02 cells, $\mathrm{COX}-2 / \mathrm{PGE}_{2}$ blockade significantly augmented the capacity of gemcitabine to potentiate ICD, phenocopying that of the G69 bladder cancer model (Fig. 3i, j). Difference in tumor volume post-engraftment became statistically significant
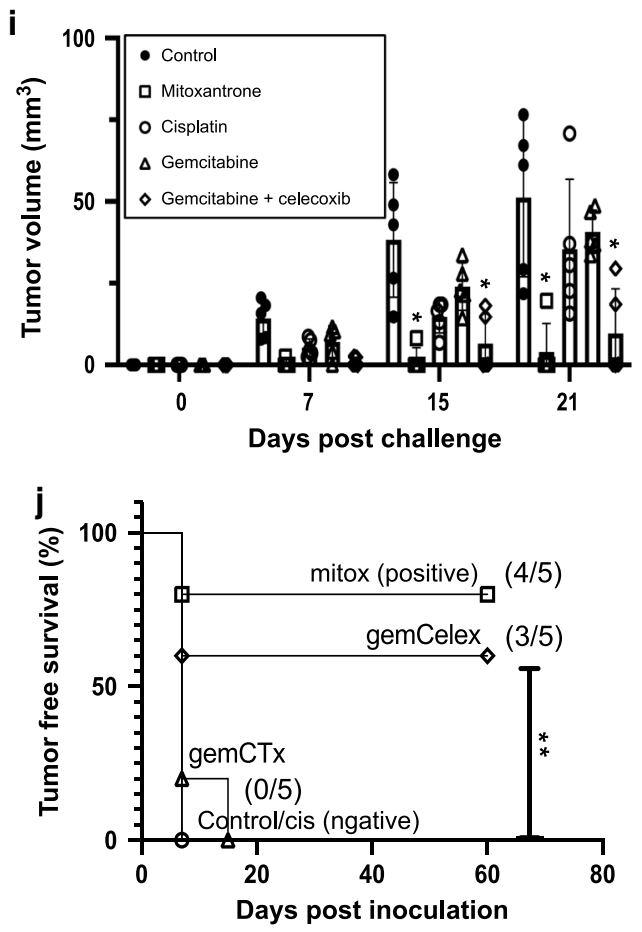

by day 15 post-challenge (Fig. 3i; $p<0.05 ; n=5$ mice per group). And as expected, vaccination using mitoxantrone resulted in the greatest tumor rejection (4/5), while the control (PBS), cisplatin and gemcitabine groups resulted poorly (0/5; Fig. 3j). Corroborating our findings in G69 (Fig. 3g, h), the gemCelex vaccine group using Panc02 also displayed enhanced tumor rejection (3/5; Fig. 3j). These results support 
Fig. 3 Prostaglandin $\mathbf{E}_{\mathbf{2}}$ release hinders ICD-induction by gemcitabine. a Western blot analysis of COX-2 and corresponding ELISA of PGE 2 from G69 cells treated with gemcitabine in vitro (representative plot shown with two technical replicates of $n=3$ independent experiments). b A schematic depicting the proposed interventional strategy that exploits the immunostimulatory/inhibitory DAMP balance to harness immunogenic cell death. $\mathbf{c}$, $\mathbf{d}$ Flow cytometry of cell surface CRT and HSP70 of single cells (example gating depicted in Supplementary Fig. 3), as well as western blot of HMGB1 from G69 and $\mathrm{PancO} 2$ cells treated with gemcitabine plus celecoxib to achieve iDAMP blockade (representative plot shown with two technical replicates of $n=3$ independent experiments). e, $\mathbf{f}$ Western blot of COX-2 and ELISA of PGE 2 from murine $\mathrm{G} 69$ and Panc02 cells to demonstrate the efficacy of celecoxib in abrogating gemcitabine-induced $\mathrm{PGE}_{2} /$ iDAMP release in vitro (representative plot shown with two technical replicates of $n=3$ independent experiments). $\mathbf{g}$ Temporal changes in tumor volume and $\mathbf{h}$ tumor-free survival of mice after challenge ( $n=9$ per treatment group) resulting from a gold-standard vaccination assay. $\mathbf{i}$ Temporal changes in tumor volume and $\mathbf{j}$ tumor-free survival of mice after challenge ( $n=5$ per treatment group) resulting from a goldstandard vaccination assay using the murine Panc02 PDAC model. celex celecoxib, CTx chemotherapy, gem gemcitabine. Statistics: two-tailed, unpaired T test (a; $p=0.0107)$; two-tailed, one-way ANOVA-Tukey's multiple comparisons test $(\mathbf{c} ; p=0.002$ [top] and $p=0.008$ [bottom]), (d; $p=0.0011$ [top] and $p=0.0007$ [bottom]), (e; ${ }^{\star *} p=0.0076$ and $\left.{ }^{* \star} p=0.0028\right),(\mathbf{f} ; p=0.0033),\left(\mathbf{g}\right.$; ${ }^{* \star} p=0.015$ and $\left.{ }^{* \star *} p=0.0003\right)$, and (i; $\left.{ }^{*} p<0.0486\right) ;$ Kaplan-Meier survival analysis using Mantel-Cox test $(\mathbf{h} ; p=0.0001)$ and $(\mathbf{j} ; p=0.004)$; and where appropriate, data are presented as mean values \pm SEM.

the notion that ICD is governed by an intricate balance between immunostimulatory and inhibitory DAMP, which is generalizable in different tumor types. Current strategies focus on substantiating immunostimulatory DAMP release to potentiate $\mathrm{ICD}^{22}$ without considering the existence or manipulation of inhibitoy DAMP release, underscoring the distinctiveness of the current approach.

An inhibitory signal 0 counteracts immunostimulatory DAMPs to mitigate immunogenic dendritic cell maturation. Dendritic cells (DCs) are quintessential professional antigen-presenting cells that excel at antigenic crosspresentation and cross-priming of $\mathrm{CD}^{+} \mathrm{T}$ cells ${ }^{35,36}$. And thus, the vaccination assay is conceptualized to rely on the successful activation/maturation of DCs for mobilizing antitumoral CD8 ${ }^{+}$ $\mathrm{T}$ cells to reject tumor engraftment ${ }^{26,27}$. Growing evidence suggests that the specialized CD103+ classical DC 1 (cDC1) subset is adept at antigenic cross-presentation and $\mathrm{CD}^{+} \mathrm{T}$ cell crosspriming 37-40 Here, we implemented bone marrowderived $\mathrm{CD}_{103}{ }^{+} \mathrm{DC}\left(\mathrm{CD}_{103}{ }^{+} \mathrm{BMDC}\right)$ cultures as a pertinent model for mechanistic study. $\mathrm{CD}_{103}{ }^{+}$BMDCs were generated from 8 -week-old mice bone marrow as previously described ${ }^{41}$. We successfully differentiated $\mathrm{CD} 11 \mathrm{c}^{+} \mathrm{CD} 103^{+} \mathrm{XCR}^{+}{ }^{+} \mathrm{MHCII}^{+}$ DCs from ex vivo bone marrow culture supplemented with fms tyrosine related kinase 3 ligand (FLT3L) and granulocytemacrophage colony-stimulating factor (GM-CSF). These DCs expressed elevated mRNA levels of basic leucine zipper ATF-Like transcription factor 3 (Batf 3 ) and interferon regulatory factor 8 (Irf8) transcription factors ${ }^{42,43}$, as well as the hallmark antigenreceptor Clec $9 a^{44}$ (Supplementary Fig. 7), confirming their characteristics as $\mathrm{CDC1}$ subset for further experimentation ${ }^{45-47}$.

Next, we assessed the immunomodulatory effects of inhibitory DAMP on $\mathrm{CD}_{103}{ }^{+}$BMDC maturation, by treating $\mathrm{CD} 103^{+}$ BMDCs with cultured media obtained from gemcitabine (gemCTx)-treated G69 cells \pm COX-2/PGE 2 blockade (Fig. 4a). COX-2/PGE 2 blockade was achieved through two approaches: (i) concurrent celecoxib drug treatment together with gemcitabine or (ii) monoclonal $\mathrm{PGE}_{2}$ neutralizing antibody to sequester $\mathrm{PGE}_{2}$ in cultured media. These $\mathrm{CD} 103^{+}$BMDC were treated with cultured media for $6 \mathrm{~h}$ and harvested for mRNA expression profiling (Fig. 4a). To evaluate the effect(s) of COX-2/PGE2 blockade on skewing $\mathrm{CD}_{103}{ }^{+} \mathrm{BMDC}$ maturation towards an immunogenic phenotype, a high-throughput qPCR-based platform (Fluidigm Biomark) and conventional qPCR were independently employed to analyze the cultured media-treated $\mathrm{CD} 103^{+}$BMDCs.

COX-2/PGE 2 blockade skewed $\mathrm{CD}_{103}{ }^{+}$BMDCs toward an immunogenic phenotype ${ }^{48}$ (Fig. $4 \mathrm{~b}$ ): confirmed by elevated mRNA expression of (i) H2-k (i.e., MHCI); (ii) canonical DC co-stimulatory receptor $C d 40^{49}$; and (iii) T-cell polarizing cytokines Tnfa, Il-12b, and Ifng ${ }^{50}$ (Fig. 4b, c). In contrast,
CD103+ BMDCs without COX-2/PGE 2 blockade exhibited a tolerogenic phenotype ${ }^{48,51}$ (Fig. 4b), characterized by the expression of: (i) Arg1, a gene important in diminishing $\mathrm{T}$ cell activity $^{52,53}$; (ii) Ido1, a gene important in preventing $\mathrm{T}$ cell expansion/memory formation ${ }^{54,55}$; (iii) Pdcd1lg, a gene important form promoting $\mathrm{T}$ cell anergy 56,57 ; and (iv) Tim3, an inhibitory receptor on dendritic cells ${ }^{58,59}$. However, other immunosuppression associated genes-e.g., inhibitory receptor Ctla4 and inhibitory cytokine $\mathrm{Il}$-10-were unaffected (Fig. 4c).

The above differential mRNA expressions (Fig. 4b, c) were further validated at the protein level using flow cytometry. At $6 \mathrm{~h}$ post cultured media treatment (where mRNA was analyzed), the protein expression of MHCI, MHCII, and CD40 in CD103+ BMDCs were only marginally higher in the COX-2/PGE ${ }_{2}-$ blockade groups (Supplementary Fig. 8a). Remarkedly, at $24 \mathrm{~h}$ post-treatment, $\mathrm{CD}_{103}{ }^{+} \mathrm{BMDCs}$ displayed significant elevation of major active marks for DCs in the COX-2/PGE 2 -blockade groups. These included MHCI, MHCII, CD40, CD86, and IL-12 protein expression (Fig. 4d and Supplementary Fig. 8b). The tolerogenic cytokine, IL-10, was significantly reduced as a result of COX-2/PGE 2 blockade (Fig. 4d). The increased protein expression of MHCI, CD40, and IL-12 (Fig. 4d and Supplementary Fig. 8b) were reflective of the increased mRNA expression observed in Fig. 4b, c. Collectively, these findings support $\mathrm{PGE}_{2}$ as a functional iDAMP (or inhibitory signal 0 ) that counteracts hallmark DAMPs in regulating the immunogenic activation/ maturation of $\mathrm{CD}_{103}+$ BMDCs.

COX-2/PGE 2 blockade promotes priming of a $\mathrm{CD8}^{+} \mathrm{Tc1}^{-}$ mediated immune response. Immunogenic cell death is functionally postulated to depend on a cytotoxic $\mathrm{CD}^{+} \mathrm{T}$ cell response ${ }^{27}$. Since our results from Fig. $4 \mathrm{~b}-\mathrm{d}$ revealed that COX-2/ $\mathrm{PGE}_{2}$ blockade favors immunogenic DC maturation, we reasoned that $\mathrm{COX}-2 / \mathrm{PGE}_{2}$ blockade would promote a $\mathrm{CD} 8^{+}$cytotoxic $\mathrm{T}$ (Tc)-1 cell polarized response. To evaluate this, we isolated and analyzed peripheral blood-circulating $\mathrm{CD}^{+} \mathrm{T}$ cells at $0,8,15$, and 21 days post-vaccination using fluorescence-activated cell sorting (FACS; Fig. 5a, b). The vaccination arms are as indicated in Fig. $5 c$ ( $n=3$ mice per group), following the immunization protocol described in Fig. 2e. The frequency of circulating $\mathrm{CD} 8^{+}$ T cells in peripheral blood was significantly elevated 8-days postvaccination in the COX-2/PGE - -blockade arm when compared to the other vaccination groups (Fig. $5 \mathrm{c}$ ). These results implicated $\mathrm{CD}^{+} \mathrm{T}$ cells as the effectors for tumor rejection observed in Fig. 3g, h.

To assess whether the increased number of circulating $\mathrm{CD}^{+}$ $\mathrm{T}$ cells associated with a Tc1-polarized immune response, we FACS-purified $\mathrm{CD}^{+} \mathrm{T}$ cells from 8-days post-vaccination (Fig. 5a, c) and analyzed for Tc1-associated gene expression using RT-qPCR ${ }^{60}$. Remarkably, $\mathrm{CD}^{+} \mathrm{T}$ cells isolated from mice 
a
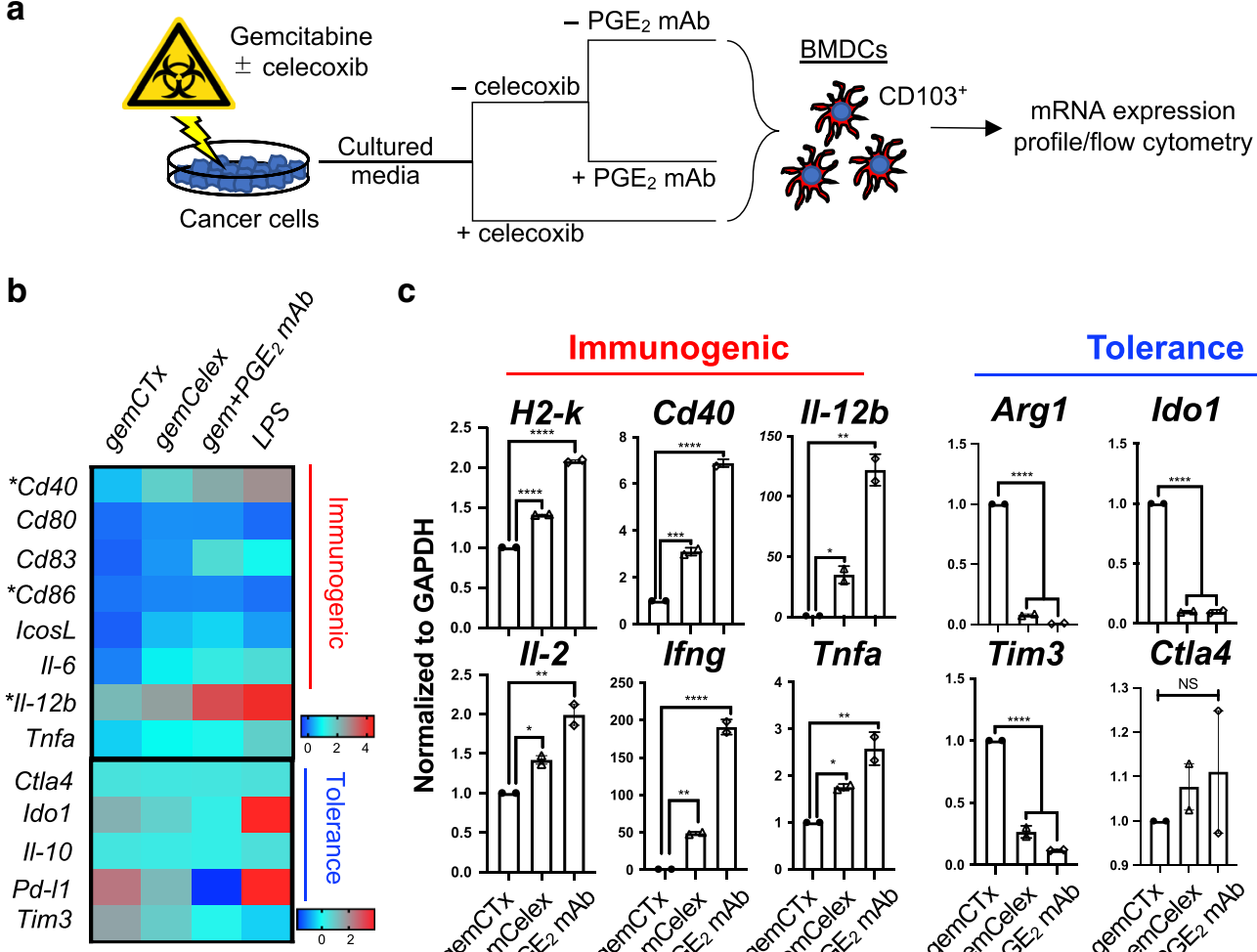

C

Log2 relative value to non-treated control DCs
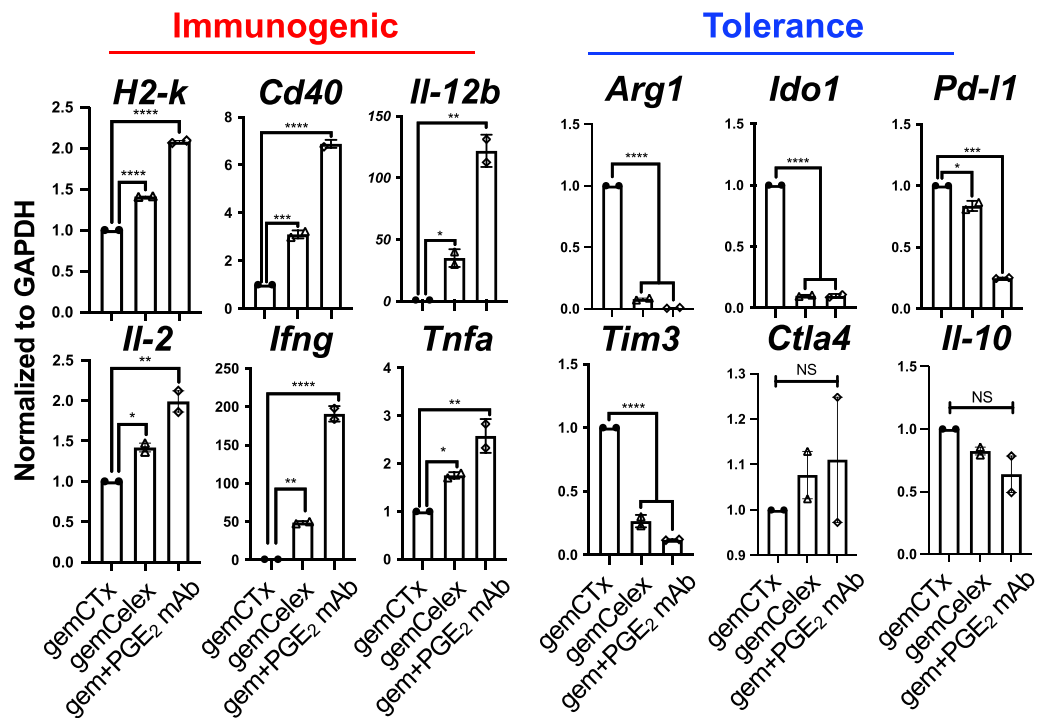

d

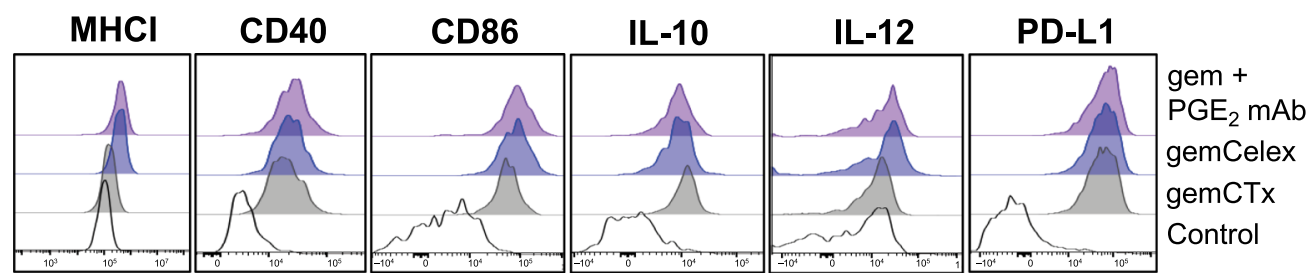

Fig. 4 An inhibitory signal 0 counteracts DAMPs to mitigate immunogenic dendritic cell maturation. a A schematic depicting the workflow utilized to assay CD103+ BMDC activation: CD103 ${ }^{+}$BMDCs were incubated with cultured media from cancer cells pre-treated with gemcitabine \pm iDAMP blockade in vitro. $\mathrm{PGE}_{2}$ neutralizing antibody or celecoxib were implemented as independent approaches to block PGE 2 action. b Heat-map derived from Fluidigm Biomark $^{\top M}$ analyzing surrogate genes representing CD103+ BMDC activity that were collected from $\mathbf{a}$. Log2 values were calculated from normalized ct values of non-treated $\mathrm{CD}_{103}{ }^{+} \mathrm{BMDCs}$ as baseline control. c qPCR validating genes associated with immunogenic versus tolerogenic dendritic cells. Relative mRNA expression was normalized to Gapdh and to gemCTx-treated CD103+ BMDCs (representative plot shown with two technical replicates of $n=3$ independent experiments). d Representative flow cytometry histogram plots of CD103 ${ }^{+}$BMDCs $24 \mathrm{~h}$ post-cultured media treatment ( $n=3$ independent experiments; example gating depicted in Supplementary Fig. 7). mAb monoclonal antibody, NS statistically non-significant. Statistics: two-tailed, one-way ANOVA-Tukey's multiple comparisons test $(H 2-k ; p<0.0001),\left(C d 40 ;{ }^{* \star *} p=0.0002\right.$ and $\left.{ }^{\star \star \star \star} p<0.0001\right),\left(I I-12 b ;{ }^{*} p=0.0475\right.$ and $\left.{ }^{\star *} p=0.0013\right) ;\left(I I-2 ;{ }^{*} p=0.323\right.$ and $\left.{ }^{* \star} p=0.0069\right)$, (Ifng; ${ }^{\star \star} p=0.0017$ and $\left.{ }^{* \star \star *} p<0.0001\right)$, (Tnfa; ${ }^{*} p=0.0347$ and $\left.{ }^{* \star} p=0.0024\right),($ Arg1; $p<0.0001)$, (Ido1; $p<0.0001),\left(P d-11 ;{ }^{*} p=0.0103\right.$ and $\left.{ }^{\star \star *} p=0.0001\right)$, (Tim3; $\left.p<0.0001\right)$; and where appropriate, data are presented as mean values \pm SEM.

injected with the COX-2/PGE 2 -blockade vaccine exhibited elevated mRNA expression of the Tc1 transcription factor $T$ bet, as well as Tcl-associated cytokines Tnfa and Infg, when compared to the other vaccination groups (Fig. 5d). Granzyme $\mathrm{B}$, a marker of cytotoxic activity, was also elevated in the same $\mathrm{CD}^{+} \mathrm{T}$ cells, illustrating COX-2/PGE 2 blockade effectively induced Tc1-effector genes.

To verify these mRNA expression findings at the protein level, we adapted the vaccination assay and developed another model where the vaccine-draining lymph nodes (vdLN; i.e., popliteal) from murine footpad injections can be collected for flow cytometry analysis. We also developed Cox $-2^{-1-}$ G69 cells using CRISPR/Cas9 as a genetic approach to definitively abrogate $\mathrm{PGE}_{2}$ biosynthesis (Supplementary Fig. 9). Appropriate vaccines (i.e., control [PBS], gemCTx, gemCelex, and gemCox $-2^{-1-}$ ) were then injected in the footpad to evaluate their relative mechanistic effects in regulating CD8 ${ }^{+} \mathrm{T}$ cell function $(n=4$ for control and $n=7$ lymph nodes per vaccination group). Five days postvaccination, the popliteal vdLN were harvested and examined for $\mathrm{CD}^{+} \mathrm{T}$ cell polarization using flow cytometry (Fig. 5a, e). Reflective of the transcriptional results in Fig. $5 \mathrm{~d}$, there was a statistically significant enrichment of T-bet positive $(p<0.05)$ and IFN $\gamma$-producing $(p<0.005) \mathrm{CD}^{+} \mathrm{T}$ cells that also displayed enhanced CD107a (an established marker for T cell activity; $p<0.005$ ) in the COX-2/PGE 2 -blocked vaccine groups (Fig. 5f, g). Intriguingly, the gemcitabine-only vaccine promoted an enrichment for Tc17/Treg polarization (Fig. 5h, i), where COX-2/PGE $2_{2}$ blockade skewed $\mathrm{CD}^{+}$T-cell polarization toward a Tc1 phenotype (Fig. 5f, g). These gemcitabine-only vaccines did not display CD107a ${ }^{+}$enrichment (Fig. 5f, g). Moreover, these T-cell 
a
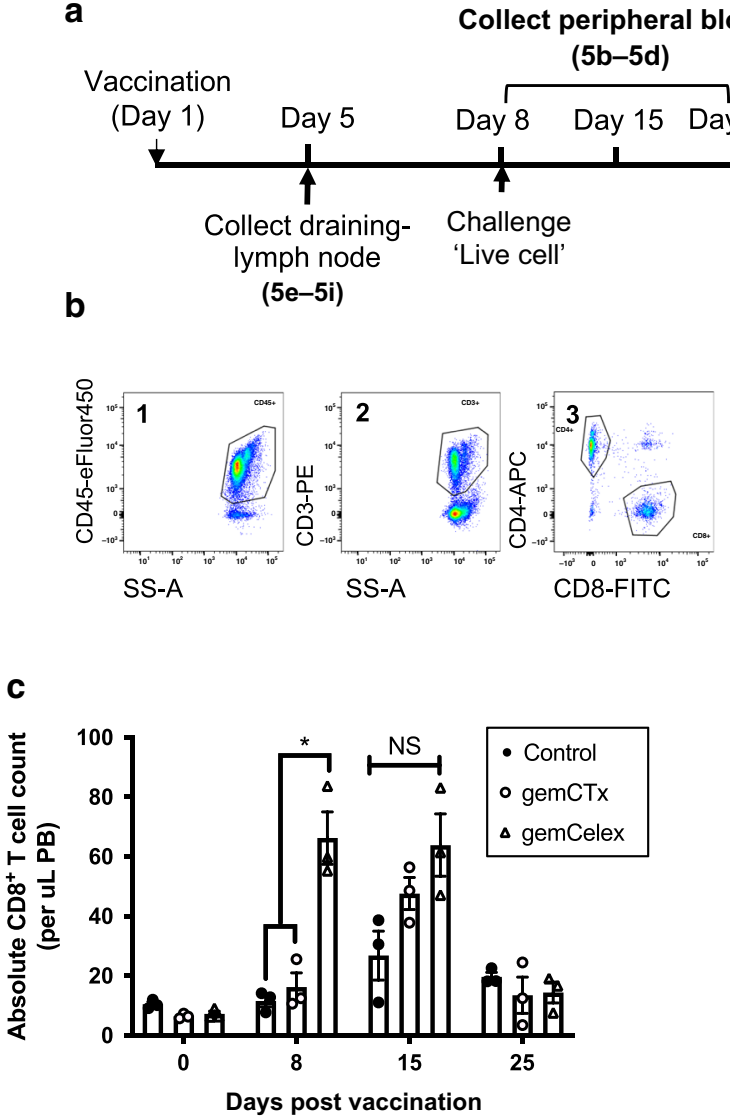

d

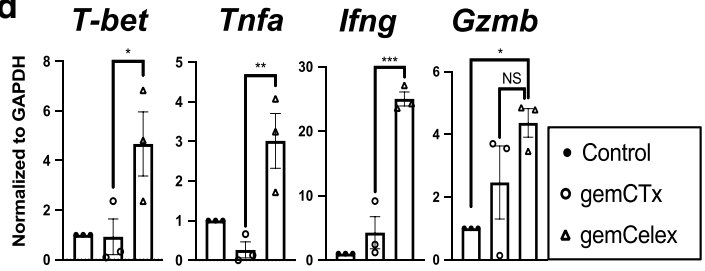

e Footpad

vaccination

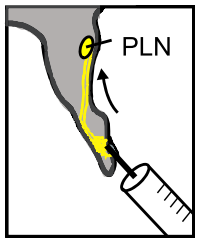

f

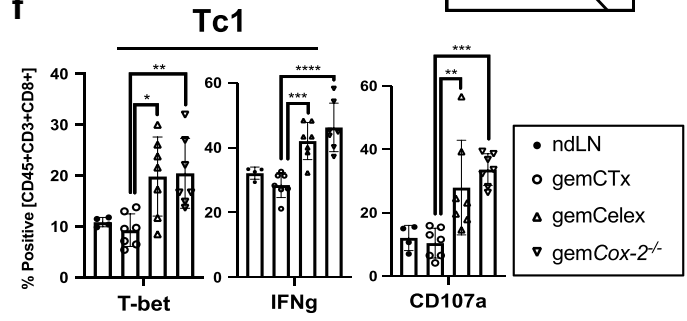

Tumor volume/ Tumor-free survival g

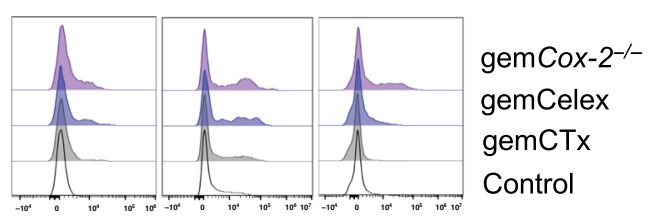

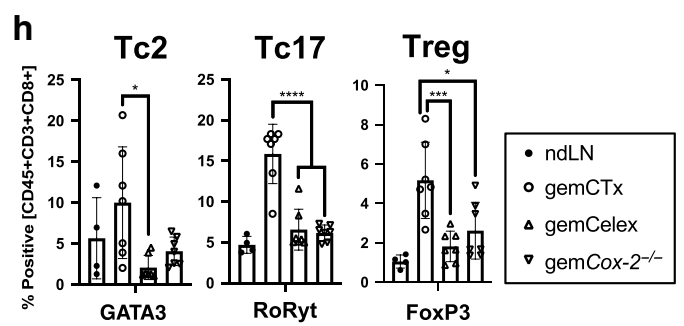

i

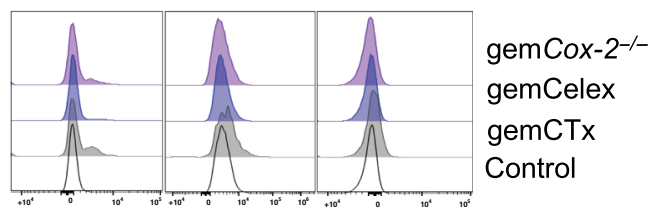

Fig. 5 COX-2/PGE 2 blockade promotes priming of a CD8 ${ }^{+}$Tc1-mediated immune response. a $A$ schematic depiction of the experimental procedures implemented to analyze vdLN (i.e., popliteal) and PB-circulating CD8 ${ }^{+} \mathrm{T}$ cells. b Representative gating-strategy implemented to analyze/sort $\mathrm{CD} 8^{+} \mathrm{T}$ cells from vdLN and PB by flow cytometry. c Quantification of PB-circulating CD $8+$ T cells post-vaccination ( $n=3$ per treatment group). $\mathbf{d}$ qPCR analysis of genes associated with functionally activated Tc1-polarized $\mathrm{CD} 8^{+} \mathrm{T}$ cells. e An illustration depicting the adapted in vivo vaccination assay to interrogate vdLN CD8 ${ }^{+}$T cells. $\mathbf{f}-\mathbf{i}$ Flow cytometry analysis and quantification of vdLN CD8 ${ }^{+}$T cells 5 -days post-vaccination ( $n$ on-dLN $n=4$; vdLN $n=5$ per group). PB peripheral blood, PLN popliteal lymph node. Statistics: two-tailed, two-way ANOVA-Tukey's multiple comparisons test (c; $\left.{ }^{*} p=0.387\right)$; two-tailed, oneway ANOVA (d, T-bet; $p=0.0483)$, (d, Tnfa; $p=0.0080)$, (d, Ifng; $p=0.0002)$, (d, Gzmb; $p=0.0379)$, (f, T-bet; ${ }^{*} p=0.0136$ and $\left.{ }^{* *} p=0.0089\right),(\mathbf{f}$,

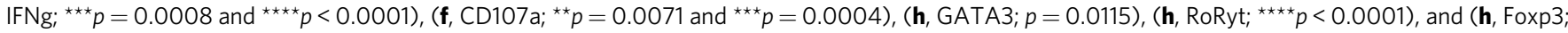
${ }^{\star} p=0.0103$ and $\left.{ }^{\star \star \star} p=0.0008\right)$; and where appropriate, data are presented as mean values \pm SEM.

polarizing effects were specific to the corresponding vdLNs; we collected the non-draining popliteal lymph nodes (ndLNs) from the opposite limbs and analysis revealed no specific polarization nor $\mathrm{T}$ cell activation (Fig. $5 \mathrm{f}-\mathrm{i}$ ). These results underscore the significance of the vaccines in promoting specific $\mathrm{T}$ cell response (s). Combined with prior results in Fig. $3 g-j$, these data implicate ICD-induced antitumoral $\mathrm{CD} 8^{+} \mathrm{T}$-cell response results from $\mathrm{T}$ cell skewing towards a Tc1 rather than a Tc17/Treg polarization type.

Immunogenic cell death-induced $\mathrm{CD8}^{+} \mathrm{T}$-cell response causes tumor rejection upon challenge. Interferon $\gamma$-secreting cytotoxic $\mathrm{CD}^{+} \mathrm{Tc1}$ cells are crucial for host protective antitumoral immunity ${ }^{61}$. Since COX-2/PGE 2 blockade favored Tc1 polarization (Fig. 5f, g), we next evaluated whether immunogenic cell death-induced tumor rejection was dependent on $\mathrm{CD}^{+} \mathrm{T}$ cell activity. To functionally interrogate this, we employed an established protocol of anti-CD8 monoclonal antibody treatment to deplete $\mathrm{CD}^{+}$cells from vaccine recipient hosts (Supplementary Fig. 10). We again implemented celecoxib (pharmacological) and Cox $-2^{-1-}$ (genetic) for COX-2/PGE 2 blockade. As expected, gemCelex and gemCox-2-1- (iDAMP blockade) vaccine groups resulted favorably with 4/8 (pharmacological) and 11/15 (genetic) mice rejecting tumor engraftment (Fig. 6a, b; statistically significant $p<0.05)$. In the presence of CD8-depletion, however, regardless of the vaccination type, all tumors engrafted and became palpable by 17 days post-challenge (Fig. 6 c, d; $n=4$ or 5 


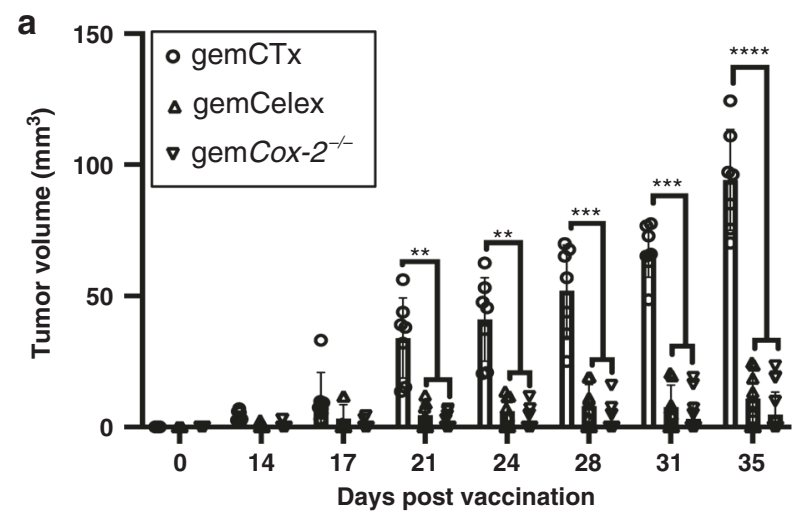

b
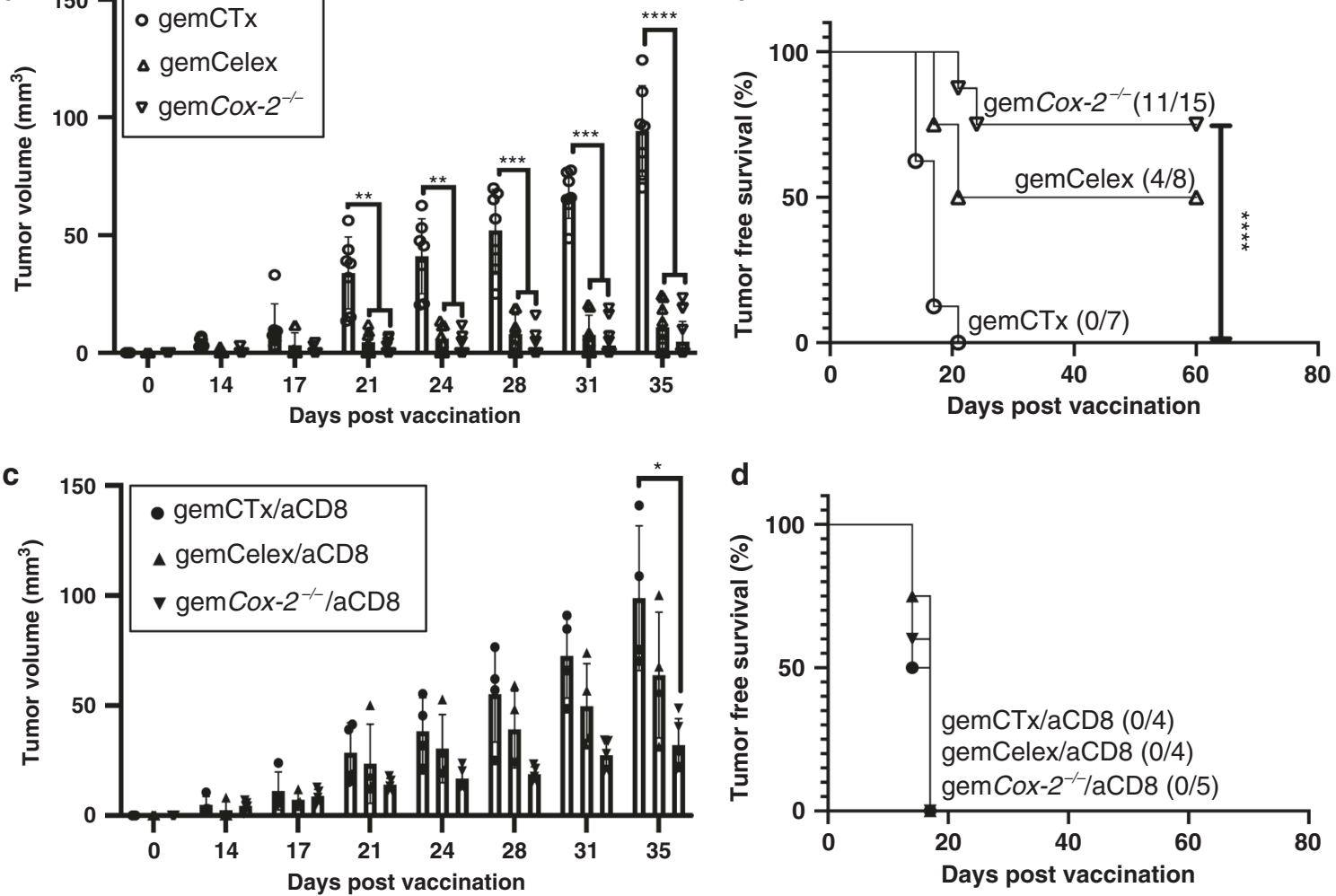

Fig. 6 CD8 ${ }^{+}$T-cell-mediated immune response drives tumor rejection upon challenge. Corresponding vaccination assay resulting from two methodologies of iDAMP blockade: pharmacological (i.e., celecoxib) and genetic (CRISPR/Cas9 KO). a Tumor volume and $\mathbf{b}$ tumor-free survival from the same vaccination assay comparing two methods to deplete $\mathrm{PGE}_{2} / \mathrm{iDAMP}$ and their efficacies in affecting drug-induced immunogenic cell death.

Corresponding aCD8 mAb treatment results as shown in c, $\mathbf{d}$ Vaccine groups: gem $(n=7)$; gemCelex $(n=8)$; gemCox-2-/- $(n=15)$; gem/aCD8 $(n=4)$; gemCelex/aCD8 $(n=4)$; gemCox- $2^{-/} / \mathrm{aCD} 8(n=5)$. Statistics: two-tailed, two-way ANOVA-Tukey's multiple comparisons test $\left(\mathbf{a} ;{ }^{\star \star} p<0.0028,{ }^{\star \star \star} p<\right.$ 0.0006 , and $\left.{ }^{\star \star \star \star} p<0.0001\right)$ and $(\mathbf{c} ; p=0.0452)$; Kaplan-Meier survival analysis using Mantel-Cox test $(\mathbf{b} ; p<0.0001)$; and where appropriate, data are presented as mean values \pm SEM.

mice per vaccination group, as indicated). Combined with the vdLN data, we demonstrate that immunogenic cell death is dependent on an intricate balance between immunostimulatory and inhibitory DAMPs that converge as signal 0 to promote a type-1-polarized $\mathrm{CD}_{103}{ }^{+} \mathrm{DC} / \mathrm{CD}^{+}{ }^{+} \mathrm{T}$ cell immune response, resulting in tumor rejection (Fig. 7).

\section{Discussion}

Gemcitabine-a standard-of-care chemotherapy that is commonly prescribed to treat various solid tumors, including bladder and pancreatic cancers-in facilitating antitumor immunity has long been debated. In the present study, we employed unbiased mass spectrometry proteomics and other molecular approaches to demonstrate that gemcitabine, as monotherapy, can indeed induce the ectopic expression of hallmark DAMPs. These findings were conserved in both human and murine models of bladder cancer, as well as a murine pancreatic ductal adenocarcinoma (PDAC) model. These results contrast a prior study reporting on the inability of gemcitabine to promote cell surface CRT expression ${ }^{28}$.

The current defining molecular feature of ICD includes the ectopic expression or release of hallmark, immunostimulatory DAMPs $^{3-5,7,31,32}$. As a result, anticancer agents that promote successful hallmark DAMP release are anticipated to initiate antitumoral immunity ${ }^{3-5,7,31,32}$. Here, we evaluated the immunogenicity of gemcitabine using independent murine bladder cancer and PDAC as study models. Our results unexpectedly revealed that gemcitabine is unable to propagate proper

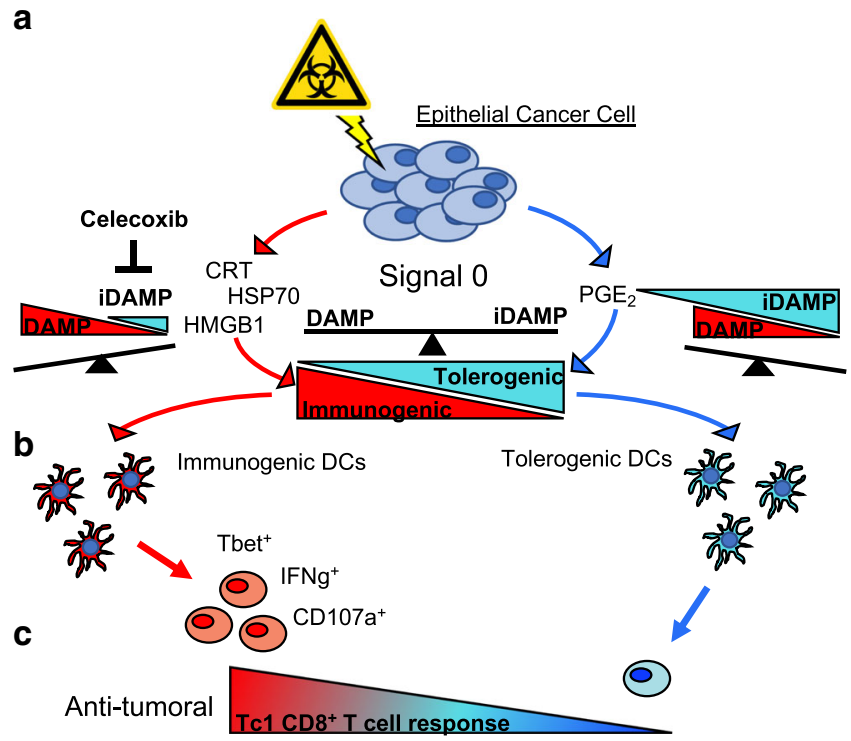

Fig. 7 schematic depiction of the proposed immunostimulatory/inhibitory DAMP balance model in modulating drug-induced immunogenic versus tolerogenic cell death fate. a The modulation of immunogenic cell death via iDAMP blockade. $\mathbf{b}$ Signal 0 skews the maturation phenotype of dendritic cells. c Resulting polarization and antitumoral $\mathrm{CD} 8^{+} \mathrm{T}$ cell response. 
antitumoral immune response in a gold-standard in vivo vaccination assay. In contrast to conventional wisdom, we found that hallmark DAMPs alone are not the only factor(s) involved in regulating the immunogenicity of drug-induced cell death.

We previously reported on gemcitabine-induced release of $\mathrm{PGE}_{2}$, a downstream metabolite derived from arachidonic acid, promotes chemoresistance via inducing the repopulation or compensatory proliferation of residual cancer stem cells ${ }^{34,62}$. Most recently, $\mathrm{PGE}_{2}$ was implicated to function as an immunomodulatory inhibitory DAMP (iDAMP) by suppressing macrophage function in the context of mechanical stress ${ }^{33}$. Our results exemplify the immunosuppressive function of this iDAMP as a physiologic response to drug-induced cell death. Furthermore, we identified a regulatory circuit that relies on the intricate balance between immunostimulatory and inhibitory DAMPs to effectively propagate ICD, culminating with antitumoral $\mathrm{CD}^{+} \mathrm{T}$ cells. We also functionally demonstrated the pharmacological applicability of tipping the DAMP/iDAMP balance by converting gemcitabine from a non-ICD-inducer into an ICD-inducing drug.

Since the gold-standard in vivo vaccination assay relies on a multimodal, stepwise cascade of immune signaling transduction, to elucidate the mechanistic details, we utilize a bone marrowderived dendritic cell culture to assess the effects of $\mathrm{PGE}_{2}$ on DC activation and maturation. Complimenting the in vivo vaccination assay, BMDCs provided a controlled in vitro system, which allowed us to interrogate the initial immunomodulatory function of dying cell-released $\mathrm{PGE}_{2}$. Importantly, our in vitro findings utilizing BMDCs correlated with in vivo data obtained from the vaccination assay, as well as the profiling of peripheral bloodcirculating and vaccine-draining lymph node $\mathrm{CD} 8^{+} \mathrm{T}$ cells. Such findings provide new insights into how cell death associated $\mathrm{PGE}_{2}$ release precludes successful propagation of an antitumoral Tc1polarized immunity, in the context of chemotherapy treatment. Clinically, these results also provide a conceptual foundation to explain why other chemotherapies are also non-ICD-inducing in addition to gemcitabine. Such concept is timely and could have important clinical implications, since many ongoing clinical trials are combining chemotherapy with immune checkpoint blockade in attempt to achieve synergistic efficacies. However, these trials have largely resulted with mixed clinical responses depending on the tumor type. In bladder carcinomas, a recent Phase 3 clinical trial combining gemcitabine-based chemotherapy regimen with immune checkpoint blockade failed to meet the expected clinical endpoint ${ }^{63}$, underscoring the clinical pertinence to evaluate $\mathrm{COX}-2 / \mathrm{PGE}_{2}$ blockade as an adjuvant therapy to enhance chemoimmunotherapeutic efficacy.

Indeed, tumor-derived $\mathrm{PGE}_{2}$ has been previously reported to promote melanoma tumor growth through immune evasion in a treatment-naive setting 64 , whereas a follow-up study further revealed that the tumor-derived $\mathrm{PGE}_{2}$ hindered $\mathrm{CD}_{103}{ }^{+} \mathrm{DC}$ recruitment into the tumor microenvironment ${ }^{65}$. Furthermore, the genetic ablation of $\mathrm{PGE}_{2}$ in melanoma was shown to synergize with PD-1 blockade to enhance antitumoral immunity ${ }^{64}$. These data corroborate the most recent studies indicating the expression of dendritic cell PD-L1 to be the key regulator of T-cell immunity in cancer ${ }^{66,67}$. In the context of chemotherapeutic treatment (i.e., current study), cell death-induced release of $\mathrm{PGE}_{2}$ also induces tolerogenic DC maturation, which will likely diminish immune checkpoint response. Since we demonstrated the effectiveness of $\mathrm{PGE}_{2}$ blockade in converting a non-ICD-inducing chemotherapy (i.e., gemcitabine) into an ICD-inducer; together with the above studies, we speculate that iDAMP (or $\mathrm{PGE}_{2}$ ) blockade will synergize with immune checkpoint blockade in the context of chemotherapy treatment. To fully evaluate the applicability of iDAMP blockade in a therapeutic context, utilization of preclinical model(s) following standard-of-care treatment regimen is recommended before its advancement into clinical trials. Thus, the functional assessment of $\mathrm{PGE}_{2}$ blockade plus chemotherapy will shed light on the immunological response(s) that result from a chronic treatment setting on pre-existing tumors-as opposed to the acute immunological response that ensue from vaccination with dying cancer cells.

Several studies ${ }^{17,21}$ recently documented on the outcome of clinical trials that evaluated the prognostic value of immunostimulatory DAMPs and their association with the therapeutic efficacy of bona fide ICD-inducing chemotherapies ${ }^{68-71}$. These reports focused on common ICD-induced immunostimulatory DAMPs as biomarkers (i.e., HMGB1 and CRT) in various cancer types, including breast and colorectal cancer, as well as AML ${ }^{68-71}$. However, based on our present findings and through the works of others $33,34,64,65$, it seems imperative to consider iDAMP as an additional biomarker; $\mathrm{PGE}_{2}$ has been reported to negatively influence the immune landscape in various tumor types 59,60 . Our findings will most definitely impact the current understanding of chemotherapy-induced ICD in the clinical setting.

Here we show that a non-ICD-inducing chemotherapy can be converted into an ICD-inducing drug through $\mathrm{PGE}_{2}$ blockade and provide new insight towards the fundamental understanding of immunogenic cell death. Previous high throughput studies only evaluated drug-induced DAMPs as an indicator to determine whether a certain drug is immunogenic or non-ICDinducing. The evidence of $\mathrm{PGE}_{2}$ as an inhibitory DAMP and the likely existence of a whole family of other iDAMPs will provide additional context to prior studies, as well as burgeon new avenues to exploit ICD in the context of immune checkpoint blockade therapies.

\section{Methods}

Cell culture. The human bladder cancer line, T24, was purchased from ATCC, and was maintained in DMEM (Sigma, D5796) supplemented with $10 \%$ fetal bovine serum (Sigma, catalog no. F0926) containing $100 \mu \mathrm{g} / \mathrm{mL}$ of streptomycin and 100 Units/mL of penicillin (GE Healthcare, SV30010). The murine bladder cancer cell line, G69, was generated by our laboratory, and was maintained in DMEM/F12 (Sigma, D6421) supplemented with $10 \%$ fetal bovine serum containing D-glucose (Fisher, AAA168280E), 1x sodium pyruvate (Gibco, 160070), 1x glutamax (Gibco, 35050061), 1x insulin-transferrin-selenium (Gibco, 41400045), $100 \mu \mathrm{g} / \mathrm{mL}$ of streptomycin, and 100 Units $/ \mathrm{mL}$ of penicillin. The Panc02 cells were maintained in DMEM high supplemented with 10\% FBS and 1\% PS. T cells and bone marrowderived dendritic cells were maintained in RPMI media (Sigma, R8758) supplemented with $50 \mu \mathrm{M}$ beta-mercaptoethanol (Santa Cruz Biotechnology, sc-202966), 1x glutamax (Gibco, 35050061), and 10\% heat-inactivated fetal bovine serum. Cells were incubated at $37^{\circ} \mathrm{C}$ in a humidified atmosphere containing $5 \% \mathrm{CO}_{2}$. In vitro treatment of cells with chemotherapy were carried out in DMEM high cultured media supplemented with reduced fetal bovine serum concentration of $2 \%$. T24, G69, and Panc02 cells were treated with appropriate IC-50 concentrations of mitoxantrone (Sigma, M6545-10MG), gemcitabine (TCI, 501332958), or cisplatin (Sigma, P4394). Celecoxib (Selleck Chemicals, 50-784-7) treatment of $3 \mu \mathrm{g} / \mathrm{mL}$ was used for all cell lines. At the time of collection, cultured media were first centrifuged at $1200 \times g$ for 5 min to ensure the collection of floating cells. Supernatants were then centrifuged at $18,000 \times g$ for $15 \mathrm{~min}$ at $4{ }^{\circ} \mathrm{C}$ to pellet cellular debris. Debris-free supernatants were utilized for downstream ELISA, ATP-Luciferase assay, and western blot analyses. Adherent cells were dissociated using TrypLE express enzyme (Gibco, 12605028), combined with the detached, floating cell pellets, and re-pelleted by centrifugation $(1600 \times g$ for $5 \mathrm{~min}$ at room temperature) for downstream flow cytometric and western blot analyses.

Mice. Wild-type FVB and C57/Blk6 mice were utilized for experimental purposes All in vivo experiments used 8- to 12-week-old mice, housed in either Baylor College of Medicine or Cedars-Sinai Medical Center animal facilities. All studies were performed in accordance with procedures approved by the Institutional Animal Care and Use Committee of Baylor College of Medicine and Cedars-Sinai Medical Center.

Gold-standard in vivo vaccination assay. G69 and Panc02 cells were seeded at $4.7 \times 10^{4}$ cells per $\mathrm{mm}^{2}$ and were treated with either cisplatin (negative control), mitoxantrone (positive control), gemcitabine, or gemcitabine plus celecoxib for 24 $\mathrm{h}$ in vitro. After $24 \mathrm{~h}$, adherent cells were washed with DPBS (Sigma, catalog no. D8537) thoroughly, dissociated with TrypLE express enzyme and pelleted by 
centrifugation $(1300 \times g$ for $5 \mathrm{~min}$ at room temperature). Cell pellets were washed with DPBS once more to ensure clearance of residual enzyme and pharmacological agent(s). $5 \times 10^{5}$ cells were suspended in $15 \mu \mathrm{L}$ of DPBS and were injected either (i) subcutaneously into the left lower flank or $i i)$ footpad of mice. A week following vaccination (lower flank vaccination), mice were challenged with $5 \times 10^{5}$ cells that were suspended in $15 \mu \mathrm{L}$ of DPBS. Mice vaccinated in the footpad were euthanized 5 days post-vaccination and the vaccine-draining lymph nodes (i.e., popliteal) were collected for subsequent analysis. Tumor growth and incidence were recorded twice a week with calipers. Peripheral blood was taken from mice at days $0,8,15$, and 22 post-vaccination. Tumor volume was calculated using the standard formula (width $\times$ length $\times$ length $/ 2$ ).

CD8 ${ }^{+}$cell depletion. Mice were injected subcutaneously with $\mathrm{aCD} 8 \mathrm{mAb}$ (BioXcell, BE0004-1-A005) at a concentration of $200 \mu \mathrm{g}$ per $30 \mathrm{mg}$ mouse. Mice were injected twice with the aCD8 mAb prior to vaccination: first and second injections were 72 and $48 \mathrm{~h}$ prior to live cell challenge, respectively. Efficacy of CD8-depletion was checked prior to challenge using murine tail-vain blood sampling coupled with flow cytometry analysis.

Processing peripheral blood for flow cytometry. Peripheral blood from mice were taken $(40-60 \mu \mathrm{L})$ via tail-vein and collected in $1.5 \mathrm{~mL}$ BD Microtainer tubes (BD, BD365974). In all, $40 \mu \mathrm{L}$ of peripheral blood was taken from each sample and subjected to centrifugation at $875 \times g$ for $10 \mathrm{~min}$ at $4^{\circ} \mathrm{C}$ to separate plasma and cells. Red blood cells were lysed using ACK buffer (Lonza, 10548E), and remaining cells were subjected to antibody staining for flow cytometric analyses.

Processing vaccine-draining lymph nodes for flow cytometry. Vaccine-draining lymph nodes were harvested 5 days post-vaccination of murine foot pad. Nondraining lymph nodes (i.e., opposite foot) were harvested as controls. Harvested lymph nodes were passed through a $70-\mu$ mesh filter. Flow-through immune cells were then centrifuged and incubated in 96-well U-bottom plates coated with aCD3 (BD, 553057) and $\mathrm{aCD} 28$ (Thermo, 16-0281-82) with RPMI (50uM BME, 10\% heat-inactivated FBS, and $1 \mathrm{x}$ activation cocktail) for $6 \mathrm{~h}$. One hour prior to collection, CD107a-PE/Dazzle antibody (diluted to 1:200) was added. T cells were then collected, washed with DPBS, and processed for flow cytometric analysis.

Generation of bone marrow-derived $\mathrm{CD}_{103}{ }^{+}$dendritic cells. Generation of bone marrow-derived $\mathrm{CD}_{103}{ }^{+}$dendritic cells followed a stepwise protocol previously described by Mayer et al. ${ }^{41}$. In short, bone marrow cells were collected from murine femur and tibia. In all, $15 \times 10^{6}$ bone marrow cells were cultured in RPMI media (Sigma, R8758) supplemented with $50 \mu \mathrm{M}$ beta-mercaptoethanol (Santa Cruz Biotechnology, sc-202966), 10\% heat-inactivated fetal bovine serum, $200 \mathrm{ng} / \mathrm{mL}$ of FLT3L (PeproTech, 50399689), and $5 \mathrm{ng} / \mathrm{mL}$ of GM-CSF (Miltenyi,130-094-043) for 9 days. On day $6,5 \mathrm{~mL}$ of fully supplemented media was added to cultures. On day 9 , $3 \times 10^{6}$ floating cells were re-seeded in a new $10-\mathrm{cm}$ dish with fresh, fully supplemented RPMI culture media and were incubated at $37^{\circ} \mathrm{C}$ in a humidified atmosphere containing $5 \% \mathrm{CO}_{2}$ for an additional 7 days.

Treating CD103 + BMDCs with cultured media. CD103+ BMDCs from 16 days of culture were harvested using $5 \mu \mathrm{M}$ EDTA solution. $1 \times 10^{4}$ cells were suspended in gemCTx pre-treated G69 cultured media ( $20 \%$ of total volume and $80 \%$ base DC media as described above) in the absence or presence of iDAMP blockade. Cultured media $\mathrm{PGE}_{2}$ sequestration was achieved by treating the cultured media with $100 \mathrm{nM}$ of anti-PGE 2 mAb (Cayman, 10009814) for at least $30 \mathrm{~min}$ on ice. $\mathrm{CD}^{-} 3^{+}$ BMDCs were cultured and collected at 6 - and 24 -h post activation with appropriate culture medium treatment.

Flow cytometry/FACS. All immune cell samples were suspended in $50 \mu \mathrm{L}$ of antiCD16/32 antibody (BD, BDB553141) solution at a dilution of 1:200 in PBS for $10 \mathrm{~min}$ on ice prior to subsequent immunophenotype staining. Immunophenotype staining was performed with antibodies diluted to 1:100 (final concentration). Peripheral blood-circulating cells were stained with the following antibodies: CD45-eFluor 450 (Fisher, 501129701); CD3-PE (Biolegend, 100206); CD4-APC (VWR, NC1556315); and CD8-FITC (Biolegend, 100706). Liquid counting BEADs (335925, BD) were used to quantify the absolute count of peripheral bloodcirculating $\mathrm{CD}^{+} \mathrm{T}$ cells. Vaccine-vdLN T cells were stained with the following antibodies: CD45-Pacific Blue (Biolegend, 103126), CD3-PE (Biolegend, 100206), CD4-APC (VWR, NC1556315), CD8-FITC (Biolegend, 100706), Tbet-BV711 (Biolegend, 644819), GATA3-PerCP/Cy5.5 (Biolegend, 653812), RoRyt-PerCP/ eFluor710 (Thermo 46-6981-82), FoxP3-BV421 (Biolegend, 126419), IFNg-BV785 (Biolegend, 505838), and CD107a-PE/Dazzle (Biolegend, 121624). Intracellular staining was performed using the BD Cytofix/Cytoperm (BD, 554714) following the manufacturer's protocol. Bone marrow-derived $\mathrm{CD}_{103}{ }^{+}$dendritic cells were stained with either: CD45-eFluor 450 (Fisher, 501129701); B220-APC (Biolegend, 103212); CD11c-PE/Cy7 (Biolegend, 117318); MHCII-BV510 (Fisher, 50402975); XCR1-BV650 (Biolegend, 148220) and CD103-BV786 (BD, BDB564322), or CD45-BV570 (Biolegend, 103135), CD11c-Biotin (Biolegend, 117304), CD11bAPCR700 (BD, BD564985,), CD103-BV711 (Biolegend, 121435), XCR1-BV650
(Biolegend, 148220), H2Kq-AF647 (Biolegend, 115106), MHCII-BV510 (Biolegend, 107636), CD40-FITC (Biolegend, 124608), CD86-PerCP/Cy5.5 (Biolegend, 105028), IL-10-BV421 (Biolegend, 505022), IL-12-PE (Biolegend, 505204), PD-L1 PE/Dazzle (Biolegend, 124324), Streptavidin-PE/Cy5 (Biolegend, 405205), and Live/Dead-NearIR stain (Thermo, L10119). To determine cell-surface DAMP expression, chemotherapy-treated cancer cells were stained with anti-CRT-PE (Cell Signaling, 19780 S) or anti-HSP70-PE (Miltenyi, 130-105-549), using a final concentration of 1:100 and 1:10, respectively, as recommended in the manufacturer's instructions. Concurrently, these DAMP-stained cells were labeled with a cell viability dye (e.g., DAPI at a $1-\mu \mathrm{g} / \mathrm{mL}$ concentration) for dead cell exclusion. Al antibody cocktails were diluted in ice-cold PBS. Cells were incubated with the appropriate antibody cocktails on ice for roughly $20 \mathrm{~min}$ and washed thoroughly with ice-cold DPBS. Flow cytometric analysis of immune cells was performed on BD LSRFortessa $\mathrm{a}^{\mathrm{mw}}$ and Cytek ${ }^{\mathrm{mw}}$ Northern Lights cell analyzer. FACS was performed on BD Aria ${ }^{\text {TI }}$ II. Flow cytometric analysis of cell surface CRT and HSP70 was performed on BD LSRFortessa ${ }^{\text {Tx }}$ and BD Accurit ${ }^{\text {me }}$ C6. All flow cytometry data were processed using FlowJo software v.10.7.1

ATP detection assay. ATP release by cancer cells was performed according to the manufacturer's protocol (Abcam, catalog no. ab113849).

ELISA. ELISA analysis of PGE2 (Cayman) release by cancer cells was performed according to the manufacturer's protocol.

Western blot. Cell pellets were lysed in RIPA buffer (Emd Millipore, 20-188) with complete protease (Sigma, 11836153001) and phosphatase (Sigma, 04906837001) inhibitor cocktails. Lysates were collected by centrifugation, $18,000 \times g$ for $15 \mathrm{~min}$ at $4{ }^{\circ} \mathrm{C}$. Protein concentration was measured using Bradford BCA colorimetric assay (Bio-Rad, 5000006). In all, $15 \mathrm{mg}$ of sample lysates were subjected to western blot analysis using 10\% Tris-Glycine gel under reducing conditions. Proteins were transferred onto PVDF membranes (Emd Millipore, IPVH00010) and probed with the following primary antibodies: COX-2 [74 kDa] at 1:1000 (Cell Signaling, 12282S); GAPDH [ 37 kDa] at 1:2000 (Santa Cruz biotechnology, SC-32233); and HMGB1 [ 29 kDa] at 1:1000 (Biolegend, 651402). Secondary antibodies were purchased from the following sources: anti-mouse-HRP at 1:10,000 (Boster, BA1075) and anti-rabbit-HRP at 1:10,000 (Cell Signaling, 7074 S). Western blot bands were visualized using enhanced chemiluminescence system (Thermo, 32106) on autoradiography films (Genesee, 30-507) or the iBright ${ }^{\text {tw }}$ CL750 systems. Western blot was quantified using the ImageJ software (ver. 1.5i) or the iBright analysis software (ver. 1.5. 0).

Analysis of mRNA expression. mRNA from peripheral blood-circulating CD8 ${ }^{+}$ $\mathrm{T}$ cells and cancer cell cultured media-treated $\mathrm{CD}_{103}{ }^{+}$dendritic cells were extracted using RNA direct lysis buffer containing $10 \mathrm{mM}$ Tris-HCl pH 7.4 (Sigma, T2194), 0.25\% Igepal CA-630 (Sigma, I8890), and $150 \mathrm{mM} \mathrm{NaCl}$ (Sigma, S5886), which was freshly prepared on the day of experimentation. cDNA was synthesized using Qscript XLT cDNA supermix (Quantabio, 95161-500), in accordance with the manufacturer's protocol. Synthesized cDNAs were then subjected to preamplification using the Ssoadvanced PreAMP master mix (Bio-Rad, 1725160), in accordance with the manufacturer's protocol. Fluidigm Biomark analysis was performed using the reagents and cassette in accordance to the recommendations made by the manufacturer. Quantitative real-time PCR was performed using iTaq Universal SYBR Green Supermix (Bio-Rad, 172-5121) and Roche LightCycler96 machine. The relative abundance of mRNA was normalized to GAPDH. Primers for $\mathrm{qPCR}$ are included in supplementary table 1 .

Mass spectrometry proteomics. Parallel reaction monitoring (PRM) was implemented to detect cell-surface and extracellular DAMPs (i.e., from conditioned medium). Cell-surface proteins were obtained from T24 and G69 cells treated with or without gemCTx for $48 \mathrm{~h}$ through mechanical cell lysing, using a dounce homogenizer and subsequent separation via differential centrifugation (i.e., fractionation). The conditioned medium from T24 and G69 cells were collected at $48 \mathrm{~h}$ with or without gemCTx treatment, centrifuged to pellet cellular debris $(18,000 \times g$ for $10 \mathrm{~min}$ at $4{ }^{\circ} \mathrm{C}$ ), and subsequently subjected to mass spectrometric analyses after concentration. We utilized the PRM method using Orbitrap Fusion ${ }^{\mathrm{TN}}$ Tribrid $^{\mathrm{TM}}$ mass spectrometer. Results obtained were based on unique peptide availability and three or four unique peptides for each target protein was selected for PRM analysis. Preselected precursor ions were scanned with a 10-min predicted elution window and isolated by quadrupole followed by collision-induced dissociation MS2 analysis For relative quantification, the raw spectrum file was crunched to. mgf format by PD1.4 and then imported to Skyline with raw data file. We validated each result by deleting non-identified spectrum and adjusting the AUC range.

Generation of COX-2 KO cells. G69 cells were transduced with lentiviral packed TLCV2 plasmid obtained from AddGene (AddGene, 87360) and subsequently selected using puromycin. G69.TLCV2 cells were treated with $1 \mu \mathrm{g} / \mathrm{mL}$ doxycycline (Sigma, D9891) in complete culture media $48 \mathrm{~h}$ prior to transfection. A COX-2 sgRNA (Sigma, MMPD0000032290) and tracrRNA (Sigma, TRACRRNA05N) was 
transfected into cells using RNAiMax transfection reagent (Thermo, 13778); instructions were provided by the manufacturer's protocols. Single cell clones were selected using limiting dilution and confirmed via western blot analysis.

Statistics. All data were evaluated and graphed using Prism ver. 7 and 8 software (GraphPad). Statistical comparison between control and experimental groups were performed utilizing two-tailed Student's $t$ test, one-way analysis of variance (ANOVA), two-way ANOVA with multiple comparisons test, or Kaplan-Meier survival analysis when appropriate. Quantified data shown were repeated at least three times in independent experiments (unless specified differently). Data shown are represented as mean \pm SEM. $P$-value $<0.05$ was considered statistically significant.

Reporting summary. Further information on research design is available in the Nature Research Reporting Summary linked to this article.

\section{Data availability}

The mass spectrometry data for protein identification have been deposited via the MASSIVE repository (MSV000086386) to the Proteome X change Consortium (http:// proteomecentral.proteomexchange.org/) with the data identifier PXD022253, and data using AB Sciex 5600 instrument has been deposited to the Massive repository with identifier MSV000086407 (https://massive.ucsd.edu). The other relevant data supporting the findings in this study are available in the article, Supplementary Information, or from the corresponding author upon reasonable request.

Received: 15 May 2019; Accepted: 4 November 2020;

Published online: 07 December 2020

\section{References}

1. Nagata, S., Hanayama, R. \& Kawane, K. Autoimmunity and the clearance of dead cells. Cell https://doi.org/10.1016/j.cell.2010.02.014 (2010).

2. Green, D. R., Oguin, T. H. \& Martinez, J. The clearance of dying cells: table for two. Cell Death Differ. https://doi.org/10.1038/cdd.2015.172 (2016).

3. Galluzzi, L., Buqué, A., Kepp, O., Zitvogel, L. \& Kroemer, G. Immunogenic cell death in cancer and infectious disease. Nat. Rev. Immunol. https://oi.org/ 10.1038/nri.2016.107 (2016).

4. Yatim, N., Cullen, S. \& Albert, M. L. Dying cells actively regulate adaptive immune responses. Nat. Rev. Immunol. https://doi.org/10.1038/nri.2017.9 (2017).

5. Kroemer, G., Galluzzi, L., Kepp, O. \& Zitvogel, L. Immunogenic cell death in cancer therapy. Аnnu. Rev. Immunol. https://doi.org/10.1146/annurevimmunol-032712-100008 (2013).

6. Green, D. R., Ferguson, T., Zitvogel, L. \& Kroemer, G. Immunogenic and tolerogenic cell death. Nat. Rev. Immunol. https://doi.org/10.1038/nri2545 (2009).

7. Galluzzi, L. et al. Consensus guidelines for the definition, detection and interpretation of immunogenic cell death. J. Immunother. Cancer https://doi. org/10.1136/jitc-2019-000337 (2020).

8. Obeid, M. et al. Calreticulin exposure dictates the immunogenicity of cancer cell death. Nat. Med. https://doi.org/10.1038/nm1523 (2007).

9. Massé, D. et al. Increased expression of inducible HSP70 in apoptotic cells is correlated with their efficacy for antitumor vaccine therapy. Int. J. Cancer https://doi.org/10.1002/ijc.20249 (2004).

10. Apetoh, L. et al. Toll-like receptor 4-dependent contribution of the immune system to anticancer chemotherapy and radiotherapy. Nat. Med. https://doi. org/10.1038/nm1622. (2007)

11. Banchereau, J. \& Steinman, R. M. Dendritic cells and the control of immunity. Nature https://doi.org/10.1038/32588 (1998).

12. Tang, D., Kang, R., Coyne, C. B., Zeh, H. J. \& Lotze, M. T. PAMPs and DAMPs: Signal 0s that spur autophagy and immunity. Immunol. Rev. https:// doi.org/10.1111/j.1600-065X.2012.01146.x (2012).

13. Fang, H. et al. TLR4 is essential for dendritic cell activation and antitumor T-cell response enhancement by DAMPs released from chemically stressed cancer cells. Cell. Mol. Immunol. https://doi.org/10.1038/cmi.2013.59 (2014).

14. Medzhitov, R. \& Janeway, C. A. Decoding the patterns of self and nonself by the innate immune system. Science https://doi.org/10.1126/science.1068883 (2002).

15. Gordon, S. Pattern recognition receptors: Doubling up for the innate immune response. Cell https://doi.org/10.1016/S0092-8674(02)01201-1 (2002).

16. Wang, Q. et al. Immunogenic cell death in anticancer chemotherapy and its impact on clinical studies. Cancer Lett. https://doi.org/10.1016/j. canlet.2018.08.028 (2018).
17. Vanmeerbeek, I. et al. Trial watch: chemotherapy-induced immunogenic cell death in immuno-oncology. OncoImmunology https://doi.org/10.1080/ 2162402X.2019.1703449 (2020).

18. Foukakis, T. et al. Immune gene expression and response to chemotherapy in advanced breast cancer. Br. J. Cancer https://doi.org/10.1038/bjc.2017.446 (2018).

19. Voorwerk, L. et al. Immune induction strategies in metastatic triple-negative breast cancer to enhance the sensitivity to PD-1 blockade: the TONIC trial. Nat. Med. https://doi.org/10.1038/s41591-019-0432-4 (2019).

20. Kwa, M. et al. Serial immunological parameters in a phase II trial of exemestane and low-dose oral cyclophosphamide in advanced hormone receptor-positive breast cancer. Breast Cancer Res. Treat. https://doi.org/ 10.1007/s10549-017-4570-4 (2018).

21. Garg, A. D. et al. Trial watch: immunogenic cell death induction by anticancer chemotherapeutics. OncoImmunology https://doi.org/10.1080/ 2162402X.2017.1386829 (2017).

22. Bezu, L. et al. Combinatorial strategies for the induction of immunogenic cell death. Front. Immunol. https://doi.org/10.3389/fimmu.2015.00187 (2015).

23. Casares, N. et al. Caspase-dependent immunogenicity of doxorubicin-induced tumor cell death. J. Exp. Med. https://doi.org/10.1084/jem.20050915 (2005).

24. Fucikova, J. et al. Human tumor cells killed by anthracyclines induce a tumorspecific immune response. Cancer Res. https://doi.org/10.1158/0008-5472. CAN-11-0950 (2011).

25. Garg, A. D. et al. A novel pathway combining calreticulin exposure and ATP secretion in immunogenic cancer cell death. EMBO J. https://doi.org/10.1038/ emboj.2011.497 (2012).

26. Humeau, J., Lévesque, S., Kroemer, G. \& Pol, J. G. Gold standard assessment of immunogenic cell death in oncological mouse models. Methods Mol. Biol. https://doi.org/10.1007/978-1-4939-8885-3_21 (2019).

27. Kepp, O. et al. Consensus guidelines for the detection of immunogenic cell death. OncoImmunology https://doi.org/10.4161/21624011.2014.955691 (2014).

28. Zhao, T. et al. Inhibition of HIF-1 $\alpha$ by PX-478 enhances the anti-tumor effect of gemcitabine by inducing immunogenic cell death in pancreatic ductal adenocarcinoma. Oncotarget https://doi.org/10.18632/oncotarget.2948 (2015).

29. Panaretakis, T. et al. The co-translocation of ERp57 and calreticulin determines the immunogenicity of cell death. Cell Death Differ. https://doi. org/10.1038/cdd.2008.67 (2008).

30. Vacchelli, E. et al. Chemotherapy-induced antitumor immunity requires formyl peptide receptor 1. Science (80-.) https://doi.org/10.1126/science. aad0779 (2015).

31. Krysko, O., Aaes, T. L., Bachert, C., Vandenabeele, P. \& Krysko, D. V. Many faces of DAMPs in cancer therapy. Cell Death Dis. https://doi.org/10.1038/ cddis.2013.156 (2013).

32. Kono, H. \& Rock, K. L. How dying cells alert the immune system to danger. Nat. Rev. Immunol. https://doi.org/10.1038/nri2215 (2008).

33. Hangai, S. et al. PGE2 induced in and released by dying cells functions as an inhibitory DAMP. Proc. Natl Acad. Sci. USA https://doi.org/10.1073/ pnas.1602023113 (2016)

34. Kurtova, A. V. et al. Blocking PGE2-induced tumour repopulation abrogates bladder cancer chemoresistance. Nature https://doi.org/10.1038/nature14034 (2015).

35. Joffre, O. P., Segura, E., Savina, A. \& Amigorena, S. Cross-presentation by dendritic cells. Nat. Rev. Immunol. https://doi.org/10.1038/nri3254 (2012).

36. Embgenbroich, M. \& Burgdorf, S. Current concepts of antigen crosspresentation. Front. Immunol. https://doi.org/10.3389/fimmu.2018.01643 (2018).

37. den Haan, J. M. M., Lehar, S. M. \& Bevan, M. J. Cd8 + but Not Cd8 Dendritic Cells Cross-Prime Cytotoxic T Cells in Vivo. J. Exp. Med. https:// doi.org/10.1084/jem.192.12.1685 (2002).

38. Pooley, J. L., Heath, W. R. \& Shortman, K. Cutting edge: intravenous soluble antigen is presented to CD4 T cells by CD8- dendritic cells, but crosspresented to $\mathrm{CD} 8 \mathrm{~T}$ cells by $\mathrm{CD} 8{ }^{+}$dendritic cells. J. Immunol. https://doi.org/ 10.4049/jimmunol.166.9.5327 (2014).

39. Bedoui, S. et al. Cross-presentation of viral and self antigens by skin-derived $\mathrm{CD} 103^{+}$dendritic cells. Nat. Immunol. https://doi.org/10.1038/ni.1724 (2009).

40. Del Rio, M. L., Bernhardt, G., Rodriguez-Barbosa, J. I. \& Förster, R. Development and functional specialization of CD103+ dendritic cells. Immunol. Rev. https://doi.org/10.1111/j.0105-2896.2009.00874.x (2010).

41. Mayer, C. T. et al. Selective and efficient generation of functional Batf3dependent $\mathrm{CD}_{103}{ }^{+}$dendritic cells from mouse bone marrow. Blood https:// doi.org/10.1182/blood-2013-12-545772 (2014).

42. Hildner, K. et al. Batf3 deficiency reveals a critical role for CD8 $\alpha+$ dendritic cells in cytotoxic T cell immunity. Science (80-.) https://doi.org/10.1126/ science.1164206 (2008).

43. Sichien, D. et al. IRF8 transcription factor controls survival and function of terminally differentiated conventional and plasmacytoid dendritic cells, respectively. Immunity https://doi.org/10.1016/j.immuni.2016.08.013 (2016). 
44. Guilliams, M. et al. Dendritic cells, monocytes and macrophages: a unified nomenclature based on ontogeny. Nat. Rev. Immunol. https://doi.org/10.1038/ nri3712 (2014)

45. Jongbloed, S. L. et al. Human CD141 + (BDCA-3) + dendritic cells (DCs) represent a unique myeloid DC subset that cross-presents necrotic cell antigens. J. Exp. Med. https://doi.org/10.1084/jem.20092140 (2010).

46. O’Keeffe, M., Mok, W. H. \& Radford, K. J. Human dendritic cell subsets and function in health and disease. Cell. Mol. Life Sci. https://doi.org/10.1007/ s00018-015-2005-0 (2015).

47. Mildner, A. \& Jung, S. Development and function of dendritic cell subsets. Immunity https://doi.org/10.1016/j.immuni.2014.04.016 (2014).

48. Dalod, M., Chelbi, R., Malissen, B. \& Lawrence, T. Dendritic cell maturation: functional specialization through signaling specificity and transcriptional programming. EMBO J. https://doi.org/10.1002/embj.201488027 (2014)

49. Ma, D. Y. \& Clark, E. A. The role of CD40 and CD154/CD40L in dendritic cells. Semin. Immunol. https://doi.org/10.1016/j.smim.2009.05.010. (2009)

50. Kaiko, G. E., Horvat, J. C., Beagley, K. W. \& Hansbro, P. M. Immunological decision-making: how does the immune system decide to mount a helper Tcell response? Immunology https://doi.org/10.1111/j.1365-2567.2007.02719.x (2008).

51. Domogalla, M. P., Rostan, P. V., Raker, V. K. \& Steinbrink, K. Tolerance through education: How tolerogenic dendritic cells shape immunity. Front. Immunol. https://doi.org/10.3389/fimmu.2017.01764 (2017).

52. Bronte, V. \& Zanovello, P. Regulation of immune responses by L-arginine metabolism. Nat. Rev. Immunol. https://doi.org/10.1038/nril668 (2005)

53. Rodriguez, P. C., Quiceno, D. G. \& Ochoa, A. C. L-arginine availability regulates T-lymphocyte cell-cycle progression. Blood https://doi.org/10.1182/ blood-2006-06-031856 (2007).

54. Dai, H. \& Dai, Z. The role of tryptophan catabolism in acquisition and effector function of memory T cells. Curr. Opin. Organ Transplant. https://doi.org/ 10.1097/MOT.0b013e3282f3deel (2008).

55. Liu, Z. et al. Suppression of memory CD8 T cell generation and function by tryptophan catabolism. J. Immunol. https://doi.org/10.4049/ jimmunol.178.7.4260 (2014).

56. Chen, L. Co-inhibitory molecules of the B7-CD28 family in the control of Tcell immunity. Nat. Rev. Immunol. https://doi.org/10.1038/nri1349 (2004).

57. Tsushima, F. et al. Interaction between B7-H1 and PD-1 determines initiation and reversal of T-cell anergy. Blood https://doi.org/10.1182/blood-2006-11060087 (2007)

58. Braun, D., Longman, R. S. \& Albert, M. L. A two-step induction of indoleamine 2,3 dioxygenase (IDO) activity during dendritic-cell maturation. Blood https://doi.org/10.1182/blood-2005-03-0979 (2005).

59. Patel, J., Bozeman, E. N. \& Selvaraj, P. Taming dendritic cells with TIM-3: Another immunosuppressive strategy used by tumors. Immunotherapy https://doi.org/10.2217/imt.12.126 (2012)

60. Mittrücker, H. W., Visekruna, A. \& Huber, M. Heterogeneity in the differentiation and function of $\mathrm{CD}^{+} \mathrm{T}$ cells. Arch. Immunol. Ther. Exp. (Warsz). https://doi.org/10.1007/s00005-014-0293-y (2014).

61. Kemp, R. A. \& Ronchese, F. Tumor-specific Tc1, but not Tc2, cells deliver protective antitumor immunity. J. Immunol. https://doi.org/10.4049/ jimmunol.167.11.6497 (2001)

62. Chan, K. S. Molecular pathways: targeting cancer stem cells awakened by chemotherapy to abrogate tumor repopulation. Clin. Cancer Res. https://doi. org/10.1158/1078-0432.CCR-15-0183 (2016).

63. Galsky, M. D. et al. Atezolizumab with or without chemotherapy in metastatic urothelial cancer (IMvigor130): a multicentre, randomised, placebo-controlled phase 3 trial. Lancet https://doi.org/10.1016/S0140-6736(20)30230-0 (2020).

64. Zelenay, S. et al. Cyclooxygenase-dependent tumor growth through evasion of immunity. Cell https://doi.org/10.1016/j.cell.2015.08.015 (2015).

65. Böttcher, J. P. et al. NK cells stimulate recruitment of $\mathrm{cDC} 1$ into the tumor microenvironment promoting cancer immune control. Cell https://doi.org/ 10.1016/j.cell.2018.01.004 (2018).

66. Oh, S. A. et al. PD-L1 expression by dendritic cells is a key regulator of T-cell immunity in cancer. Nat. Cancer https://doi.org/10.1038/s43018-020-0075-x (2020).

67. Mayoux, M. et al. Dendritic cells dictate responses to PD-L1 blockade cancer immunotherapy. Sci. Transl. Med. https://doi.org/10.1126/scitranslmed. aav7431 (2020)

68. Ladoire, S., Enot, D., Andre, F., Zitvogel, L. \& Kroemer, G. Immunogenic cell death-related biomarkers: Impact on the survival of breast cancer patients after adjuvant chemotherapy. Oncoimmunology https://doi.org/10.1080/ 2162402X.2015.1082706 (2016).

69. Fahmueller, Y. N. et al. Immunogenic cell death biomarkers HMGB1, RAGE, and DNAse indicate response to radioembolization therapy and prognosis in colorectal cancer patients. Int. J. Cancer https://doi.org/10.1002/ijc.27894 (2013).

70. Fucikova, J. et al. Relevance of the chaperone-like protein calreticulin for the biological behavior and clinical outcome of cancer. Immunol. Lett. https://doi. org/10.1016/j.imlet.2017.11.006 (2018).

71. Fucikova, J. et al. Calreticulin exposure by malignant blasts correlates with robust anticancer immunity and improved clinical outcome in AML patients. Blood https://doi.org/10.1182/blood-2016-08-731737 (2016).

\section{Acknowledgements}

We thank Dr. Qizhi (Cathy) Yao for providing the Panc02 cell line used in this study; the Cytometry/Cell Sorting Cores and animal facilities at Baylor College of Medicine and Cedars-Sinai Medical Center for providing expertize and maintenance of laboratory equipment and animals; and finally, the Stehlik \& Dorfleutner Lab for providing the equipment and expertize in flow cytometry-based assays. This work was supported in part by the National Cancer Institute R00CA129640-03S1 (K.S.C.), F31CA247257 (K.H.), T32GM088129 (K.H.), CPRIT RP160283 BCM Training Program (F.N.), P30CA125123 (BCM Proteomics core) and the U.S. Department of Defense CA181002 (K.S.C.).

\section{Author contributions}

K.H. designed and conducted the experiments, analyzed and interpreted data, and prepared the manuscript; F.N., Y.C.L., and E.T. were instrumental in experiments performed and data acquisition for Fig. 3; A.V.K. collected and provided the results in Fig. 1 and Supplementary Fig. 1, and was a major editor of the current manuscript; A.J., H.E.L., and S.Y.J. were responsible for all mass spectrometry-based assays and data collected in Fig. 1 and Supplementary Fig. 1. H.G. and A.K. collected data provided in Supplemental Fig. 10; A.O. helped with manuscript edits; K.S.C. was the overseeing PI, who contributed to the overall conceptual design, coordinated the collaborations, and secured funding support for the current study.

\section{Competing interests}

The authors declare no competing interests.

\section{Additional information}

Supplementary information is available for this paper at https://doi.org/10.1038/s41467 020-19970-9.

Correspondence and requests for materials should be addressed to K.S.C.

Peer review information Nature Communications thanks the anonymous reviewers for the contribution to the peer review of this work.

Reprints and permission information is available at http://www.nature.com/reprints

Publisher's note Springer Nature remains neutral with regard to jurisdictional claims in published maps and institutional affiliations.

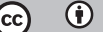

Open Access This article is licensed under a Creative Commons Attribution 4.0 International License, which permits use, sharing, adaptation, distribution and reproduction in any medium or format, as long as you give appropriate credit to the original author(s) and the source, provide a link to the Creative Commons license, and indicate if changes were made. The images or other third party material in this article are included in the article's Creative Commons license, unless indicated otherwise in a credit line to the material. If material is not included in the article's Creative Commons license and your intended use is not permitted by statutory regulation or exceeds the permitted use, you will need to obtain permission directly from the copyright holder. To view a copy of this license, visit http://creativecommons.org/ licenses/by/4.0/.

(ㄷ) The Author(s) 2020 